\title{
SUPPORT VARIETIES \\ FOR MODULES OVER CHEVALLEY GROUPS AND CLASSICAL LIE ALGEBRAS
}

\author{
JON F. CARLSON, ZONGZHU LIN, AND DANIEL K. NAKANO
}

\begin{abstract}
Let $G$ be a connected reductive algebraic group over an algebraically closed field of characteristic $p>0, G_{1}$ be the first Frobenius kernel, and $G\left(\mathbb{F}_{p}\right)$ be the corresponding finite Chevalley group. Let $M$ be a rational $G$-module. In this paper we relate the support variety of $M$ over the first Frobenius kernel with the support variety of $M$ over the group algebra $k G\left(\mathbb{F}_{p}\right)$. This provides an answer to a question of Parshall. Applications of our new techniques are presented, which allow us to extend results of Alperin-Mason and Janiszczak-Jantzen, and to calculate the dimensions of support varieties for finite Chevalley groups.
\end{abstract}

\section{INTRODUCTION}

1.1. Let $G$ be a connected reductive $k$-algebraic group defined and split over the finite field $\mathbb{F}_{p}$ of $p$ elements where $k=\overline{\mathbb{F}}_{p}$. Let $G_{1}$ be the scheme theoretic kernel of the Frobenius map. The category of $G_{1}$-modules is equivalent to the category of restricted modules for the restricted Lie algebra $\mathfrak{g}=$ Lie $G$. On the other hand, let $G\left(\mathbb{F}_{p}\right)$ be the finite (untwisted) Chevalley group obtained by taking the $\mathbb{F}_{p}$-rational points of $G$. Given a rational $G$-module over $k$ we can restrict the action to either $G_{1}$ or to $G\left(\mathbb{F}_{p}\right)$ (see Figure 1). Much effort has been made in trying to link the representation and cohomology theory for the finite group $G\left(\mathbb{F}_{p}\right)$ and the Frobenius kernel by using the ambient algebraic group. Even though the category of modules for the finite group and the Frobenius kernel share many intrinsic features, difficulties in making comparisons arise because there is no direct functorial relationship between these two categories.

In 1987, B. Parshall in an article in the Arcata Conference Proceedings entitled Cohomology of Algebraic Groups [P] stated two open problems where the first problem is a precursor to the second.

(1.1.1) If $M \in \bmod (G)$ such that $M$ is projective as a $G_{1}$-module, is $M$ projective over $G\left(\mathbb{F}_{p}\right)$ ?

(1.1.2) Let $M \in \bmod (G)$. Can we relate the support variety of $M$ for $G_{1},\left|G_{1}\right|_{M}$, with the support variety of $M$ for the finite group $\left|G\left(\mathbb{F}_{p}\right)\right|_{M}$ ?

Received by the editors April 21, 2005 and, in revised form, October 17, 2005.

2000 Mathematics Subject Classification. Primary 17B55, 20Gxx; Secondary 17B50.

The research of the first author was supported in part by NSF grant DMS-0100662 and DMS0401431.

The research of the second author was supported in part by NSF grant DMS-0200673.

The research of the third author was supported in part by NSF grant DMS-0102225 and DMS-0400548. 


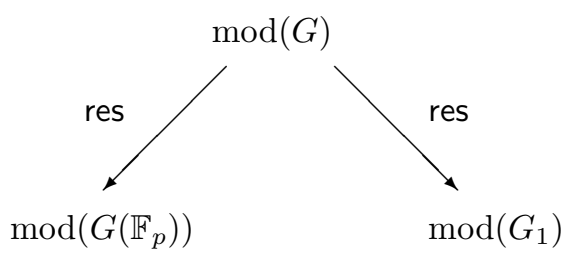

Figure 1

In $[\mathrm{LN}]$, the second and third authors showed that for $M \in \bmod (G)$,

$$
\operatorname{dim}\left|G\left(\mathbb{F}_{p}\right)\right|_{M} \leq \frac{1}{2} \operatorname{dim}\left|G_{1}\right|_{M} .
$$

This inequality was proved by developing a new approach to relate the cohomology theories of $G\left(\mathbb{F}_{p}\right)$ and $G_{1}$. Thus, Statement (1.1.1) (known as the "Parshall conjecture") is an immediate corollary of this result. Furthermore, in that paper it is shown that if $M \in \bmod (G)$ and the $G$-composition factors are $p$-bounded, then the converse to (1.1.1) also holds.

This paper is devoted to providing an affirmative answer to (1.1.2). The procedure for relating those varieties involves several new results and ideas. More precisely, through a series of works ([SFB], [FP2]) it is known that there is a $G$ equivariant isomorphism between $\left|G_{1}\right|_{k}$ and $\mathcal{N}_{1}$, where $\mathcal{N}_{1}$ is the restricted nullcone. In $[\mathrm{NPV}]$, the Jantzen conjecture on support varieties was verified. As a consequence, it follows that $\mathcal{N}_{1}=G \cdot \mathfrak{n}_{J}$ where $\mathfrak{n}_{J}$ is the nil-radical of a parabolic Lie subalgebra $\mathfrak{p}_{J}$ for some subset $J$ of simple roots when the prime is good. This answers an old question of Friedlander and Parshall on the irreducibility of $\mathcal{N}_{1}$. Recently, the authors and Parshall [CLNP] have determined representatives for the sets $J$ and have computed the dimensions of $\mathcal{N}_{1}$ (for $p$ good).

The aforementioned results are used in the following way. Let $\mathcal{U}_{1}$ be the closed subvariety of the unipotent variety $\mathcal{U}$ consisting of elements of order 1 or $p$. Note that for $p \geq h, \mathcal{U}_{1}=\mathcal{U}$ and $\mathcal{N}_{1}=\mathcal{N}$ where $\mathcal{N}$ is the ordinary nullcone. For $p$ being good for $G$, by using our explicit description of $\mathcal{N}_{1}=G \cdot \mathfrak{n}_{J}$, we verify conditions given by Seitz for the existence of an exponential map, exp $: \mathfrak{n}_{J} \rightarrow U_{J}$ $\left(U_{J}=R_{u}\left(P_{J}\right)\right.$ ) (Theorem 2). By using Springer isomorphisms between $\mathcal{N}$ and $\mathcal{U}$ and the classifications, we show that $\mathcal{U}_{1}$ is the closure of a Richardson class in $\mathcal{U}$ whose description can be immediately read off from the results in [CLNP]. We then prove that there is an extension of this map to a $G$-equivariant isomorphism, $\exp : \mathcal{N}_{1} \rightarrow \mathcal{U}_{1}$. However, we require that $\mathcal{N}_{1}$ be a normal variety. This condition is automatically satisfied when $p \geq h$. We should remark that our results also show that for $p \geq h$, the map exp can be defined on the ordinary nullcone $\mathcal{N}$. For $p$ good, Springer [Sp1] constructed a $G$-equivariant isomorphism between $\mathcal{N}$ and $\mathcal{U}$. For the purposes of this paper (i.e., for obtaining an affirmative solution to (1.1.2)) this isomorphism does not have the required properties because this map is, in general, not compatible with the restriction maps given in Figure 1. The exponential map that we construct has the important properties that it respects the group operations and it preserves the centralizers in $G$ and in $\mathfrak{g}$ (Theorem 3). 
We expect that exp is the restriction of a Springer isomorphism. This is true when $p \geq h$. If this is the case, the normality condition on $\mathcal{N}_{1}$ can be dropped.

Furthermore, our construction is compatible with Seitz's construction of the good $A_{1}$ subgroups corresponding to each unipotent element of order $p$ and provides an effective way to utilize the centralizers both in $G$ and in $\mathfrak{g}$.

The exponential isomorphism mentioned above enables us to construct maps (up to an isogeny) $\phi$ and $\psi$ between the cohomological varieties of $\mathfrak{g}$ and $G\left(\mathbb{F}_{p}\right)$ which are finite onto their images, making the diagram in Figure 2 commute (Theorem 5 ). This allows us to identify the support variety $\left|G\left(\mathbb{F}_{p}\right)\right|_{M}$ as a subvariety of $\mathcal{U}_{1}$ inside the group $G$ factored out by the action of $G\left(\mathbb{F}_{p}\right)$ (Corollary 1). Furthermore, we can also identify $\left|G\left(\mathbb{F}_{p}\right)\right|_{M}$ as a subvariety of $\left|G_{1}\right|_{M} / G\left(\mathbb{F}_{p}\right)$ for $G$-modules $M$ whose weights are not too large. In general the algebraic group $G$ does not act on $\left|G\left(\mathbb{F}_{p}\right)\right|_{M}$. Nevertheless, the existence of these maps enables us to use the rich geometric theory of nilpotent orbits in $\mathfrak{g}$ to obtain results for finite groups. Applications will be presented in the last section of the paper.

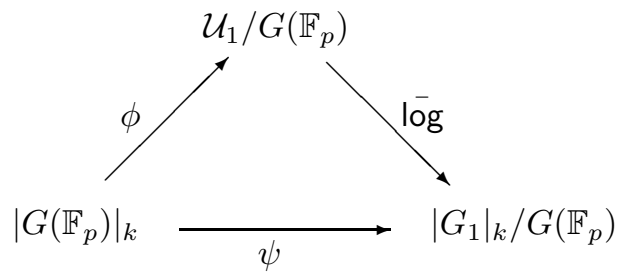

Figure 2

1.2. The paper is organized as follows. In Section 2, we apply the results of [CLNP] and $[\mathrm{NPV}]$ to extend the exponential isomorphism exp $: \mathfrak{n}_{J} \rightarrow \mathcal{U}_{J}$ to a $G$-equivariant isomorphism exp : $\mathcal{N}_{1} \rightarrow \mathcal{U}_{1}$. In Section 3, using the exponential map exp, we define the notion of $\mathbb{F}_{p}$-expressible nilpotent elements and $\mathbb{F}_{p}$-expressible unipotent elements via elementary abelian $p$-subgroups. These varieties behave well under the exponential map between the unipotent and nilpotent varieties. In Section 4, we show how to map an elementary abelian $p$-subgroup in $G\left(\mathbb{F}_{p}\right)$ into the unipotent variety using the exponential map constructed in Section 2. This will allow us to define a map, which is finite onto its image, from the cohomological variety $G\left(\mathbb{F}_{p}\right)$ into $\mathcal{U}_{1}$ factored out by the conjugation action of the finite group $G\left(\mathbb{F}_{p}\right)$. The quotient space $\mathcal{U}_{1} / G\left(\mathbb{F}_{p}\right)$ can thus be viewed as a "model" for the theory of supports for $G\left(\mathbb{F}_{p}\right)$. The logarithm map can also be used to construct a finite map from the support variety of a module for $G\left(\mathbb{F}_{p}\right)$ into $\mathcal{N}_{1} / G\left(\mathbb{F}_{p}\right)$. The images of these maps are identified at the end of the section by using our definition of $\mathbb{F}_{p^{-}}$ expressibility. Recently Friedlander and Pevtsova, [FPe, Prop. 5.8], demonstrated that the maps given above are compatible with their "support space" point of view.

In the final section, applications are provided which are both conceptual as well as computational. We indicate how earlier results of Alperin and Mason, and Janiszczak and Jantzen for finite groups $G\left(\mathbb{F}_{q}\right)$ can be further extended by using the theory of nilpotent orbits in $\mathfrak{g}$ for the groups $G\left(\mathbb{F}_{p}\right)$. Furthermore, we demonstrate how the dimensions of the support varieties of $G$-modules over finite Chevalley groups can be computed once their support varieties over $G_{1}$ are known. In particular, we compute the complexity of the simple modules for $G\left(\mathbb{F}_{p}\right)$ for all 
rank two simple groups. Also, we determine the complexity for induced modules $H^{0}(\lambda)$ over $\mathrm{GL}_{n}\left(\mathbb{F}_{p}\right)$ for $n \leq 7$ when the highest weights are not large.

A Chevalley group in this paper means an untwisted group. The twisted case is not treated in this paper. It seems as though a different approach will be necessary to prove similar results for modules over higher Frobenius kernels and $G\left(\mathbb{F}_{p^{r}}\right)$ for $r>1$.

1.3. Notation and conventions. Throughout this paper let $k$ be an algebraically closed field of characteristic $p>0$ which contains the finite field $\mathbb{F}_{p}$ of $p$ elements. Let $G$ be a simple algebraic $k$-group defined and split over the prime field $\mathbb{F}_{p}$ having root system $\Phi$ with respect to a maximal split torus $T$. Let $\Pi$ be the set of simple roots and $\Phi^{+}$(resp. $\Phi^{-}$) be the positive (resp. negative) roots. The Coxeter number of $\Phi$ is denoted by $h$. Let $B$ be the Borel subgroup containing $T$ defined by $\Phi^{-}$. Furthermore, let $X(T)$ be the integral weight lattice obtained from $T$. If $\alpha^{\vee}=2 \alpha /\langle\alpha, \alpha\rangle$ is the coroot corresponding to $\alpha \in \Phi$, then the set of dominant integral weights is defined by

$$
X(T)_{+}=\left\{\lambda \in X(T): 0 \leq\left\langle\lambda, \alpha^{\vee}\right\rangle \text { for all } \alpha \in \Pi\right\} .
$$

The Weyl group $W$ is the group generated by reflections associated to the root system $\Phi$, and $W_{p}=W \ltimes p \mathbb{Z} \Phi$ is the affine Weyl group associated to the prime $p$. Furthermore, the set of $p$-restricted weights is

$$
X_{1}(T)=\left\{\lambda \in X(T): 0 \leq\left\langle\lambda, \alpha^{\vee}\right\rangle<p \text { for all } \alpha \in \Pi\right\} .
$$

The Weyl group $W$ (affine Weyl group $W_{p}$ ) acts on $X(T)$ naturally with the "dot action" defined by $w \cdot \lambda=w(\lambda+\rho)-\rho$ where $w \in W\left(w \in W_{p}\right), \lambda \in X(T)$, and $\rho$ is the half sum of positive roots. The simple modules will be denoted by $L(\lambda)$ and the induced modules by $H^{0}(\lambda)=\operatorname{ind}_{B}^{G} \lambda$ for $\lambda \in X(T)_{+}$.

Let $\mathfrak{g}=\operatorname{Lie}(G)$ be the Lie algebra of $G$. Then $\mathfrak{g}$ is a restricted Lie algebra with $p$ th power map $[p]$. The variety of nilpotent elements of $\mathfrak{g}$ is called the nullcone and is denoted by $\mathcal{N}$. The restricted nullcone is defined as $\mathcal{N}_{1}=\left\{x \in \mathfrak{g}: x^{[p]}=0\right\}$. Let $\mathcal{U}$ be the set of unipotent elements in $G$ and let the $p$-unipotent variety $\mathcal{U}_{1}$ be defined as $\mathcal{U}_{1}=\left\{x \in G: x^{p}=1\right\}$.

Let $H$ be an affine algebraic group scheme over $k$ and let $H_{1}=\operatorname{ker}(F r)$. Here $F r: H \rightarrow H^{(1)}$ is the Frobenius map. There is a categorical equivalence between restricted Lie $(H)$-modules and $H_{1}$-modules (see [Jan1, I. Ch.9]).

For a finite $k$-group scheme $K$, the cohomology ring $R=H^{2 \bullet}(K, k)$ is a commutative, finitely generated $k$-algebra [FS]. If $M \in \bmod (K)$, the support variety of $M,|K|_{M}$, is the maximal ideal spectrum of $R / J_{M}$ where $J_{M}$ is the annihilator of $\operatorname{Ext}_{K}^{\circ}(M, M)$ in $R$. The dimension of $|K|_{M}$ can be interpreted as the complexity of the module $M[\operatorname{Ben} 1, \S 5.7]$. In this paper we will be interested in the cases when $K=G\left(\mathbb{F}_{p}\right)$ and $K=G_{1}$.

\section{An exponential map DEFined on the Restricted NullCone}

2.1. Let $R$ be a commutative ring and let $X$ be an $R$-scheme. For any homomorphism $R \rightarrow A$ of commutative rings, let $X_{A}=X \otimes_{R} A$ denote the $A$-scheme defined via base change. If $S \rightarrow R$ is a homomorphism of commutative rings, then we say that $X$ has an $S$-structure if there is an $S$-scheme $X_{S}$ such that $X=X_{S} \otimes_{S} R$. If 
$\phi: X \rightarrow Y$ is a morphism of $R$-schemes, then we denote by $\phi_{A}=\phi \otimes_{R} A: X_{A} \rightarrow Y_{A}$ the $A$-morphism of $A$-schemes. If both $X$ and $Y$ are $R$-schemes with $S$-structures $X_{S}$ and $Y_{S}$ respectively, we say the morphism $\phi$ is defined over $S$ if there is an $S$-morphism $\phi_{S}: X_{S} \rightarrow Y_{S}$ of $S$-schemes such that $\phi=\phi_{S} \otimes_{S} R$. For an $R$-scheme $X$ and any $R$-algebra $A$, we set $X(A)=\operatorname{Mor}_{R-s c h}(\operatorname{Spec}(A), X)$ to be the set of all $A$-rational points. In particular, when $X$ has an $S$-structure for a subring $S$ of $R$, we write $X(S)$ as the set of $S$-rational points of $X_{S}$ instead of $X_{S}(S)$. When $R=k$ is an algebraically closed field and $X$ is an algebraic $k$-variety, we identify $X$ with the set of all closed $k$-rational points instead of writing $X(k)$. The terminology and conventions are provided for the reader in [Jan1] and [Sp3].

Lemma 1. Let $F$ be a perfect field. Suppose that $\phi: X \rightarrow Y$ is an F-morphism of algebraic $F$-varieties and that $k$ is an algebraically closed field containing $F$ as a subfield. Then

(a) for any closed subvariety $V \subseteq Y_{k}$ defined over $F, \phi_{k}^{-1}(V) \subseteq X_{k}$ is a closed subvariety of $X_{k}$ defined over $F$, and

(b) if $\phi$ is a constant morphism of F-varieties, then so is $\phi_{k}$.

Proof. Let $\Gamma_{\phi}=\left\{(x, \phi(x)) \in X_{k} \times Y_{k} \mid x \in X_{k}\right\}$ be the graph of $\phi$. Then $\Gamma_{\phi}$ is a closed $F$-subvariety of $X \times Y$. The projections $p_{1}: X \times Y \rightarrow X$ and $p_{2}: X \times Y \rightarrow Y$ are defined over $F$ and $p_{1}: \Gamma_{\phi} \rightarrow X$ is an isomorphism (defined over $F$ ). Under this isomorphism $p_{1}$, we have $\phi^{-1}(V)$ isomorphic to $\Gamma_{\phi} \cap(X \times V)$, which is a closed $F$-subvariety of $\Gamma_{\phi}$ by $[\mathrm{Sp} 2,11.2 .13]$. This proves (a).

To see (b), we recall that $\phi_{k}$ is a constant if and only if $\phi_{k}^{*}: k[Y] \rightarrow k[X]$ factors through the embedding $k \subseteq k[X]$ (we can assume that $X$ and $Y$ are affine). It follows that $\phi_{k}$ is constant because $\phi_{F}^{*}: F[Y] \rightarrow F[X]$ factors through $F \subseteq F[X]$ and $\phi_{k}^{*}=\phi_{F}^{*} \otimes_{F} 1$.

2.2. Let $\mathfrak{g}_{\mathbb{C}}$ be a complex semisimple Lie algebra. Fix a Chevalley basis and let $\mathfrak{g}(\mathbb{Z})$ be the $\mathbb{Z}$-span of the Chevalley basis. Set $\mathfrak{g}:=k \otimes_{\mathbb{Z}} \mathfrak{g}(\mathbb{Z})$ and $\mathfrak{g}\left(\mathbb{F}_{p}\right):=\mathbb{F}_{p} \otimes_{\mathbb{Z}} \mathfrak{g}(\mathbb{Z})$. When $\mathfrak{g}_{\mathbb{C}}$ is regarded as an affine $\mathbb{C}$-variety, the Chevalley basis defines a $\mathbb{Z}$-structure $\mathfrak{g}_{\mathbb{Z}}$ on $\mathfrak{g}$. Thus $\mathfrak{g}(\mathbb{Z})$ is the set of $\mathbb{Z}$-rational points of the $\mathbb{Z}$-scheme $\mathfrak{g}_{\mathbb{Z}}$. Similarly, we use $\mathfrak{g}_{\mathbb{F}_{p}}$ to denote the $\mathbb{F}_{p}$-scheme which gives an $\mathbb{F}_{p}$-structure on the $k$-variety $\mathfrak{g}$ and use $\mathfrak{g}\left(\mathbb{F}_{p}\right)$ to denote the set of $\mathbb{F}_{p}$-rational points of $\mathfrak{g}_{\mathbb{F}_{p}}$. We now fix an algebraically closed field $k$ of characteristic $p>0$. Let $G$ be a semisimple algebraic group over $k$ with a split $\mathbb{F}_{p}$-form $G_{\mathbb{F}_{p}}$ such that $\mathfrak{g}\left(\mathbb{F}_{p}\right)$ is the Lie algebra Lie $\left(G_{\mathbb{F}_{p}}\right)$ over $\mathbb{F}_{p}$ in $\mathfrak{g}=\operatorname{Lie}(G)$. The reader is referred to [Jan1], [Bo, 18.6], and [Sp2] for further details on algebraic groups and group schemes.

2.3. Let $G$ be a semisimple algebraic group such that $G_{s c} \rightarrow G$ is separable (i.e., $\operatorname{Lie}\left(G_{s c}\right) \rightarrow \operatorname{Lie}(G)$ is surjective). Springer ([Sp1, Thm. 3.1], [SS, III, 3.12]) constructed a logarithmic map $\log _{s}: \mathcal{U} \rightarrow \mathcal{N}$ which is $G$-equivariant. Fix a regular unipotent element $u$ and regular nilpotent element $x$ such that $C_{G}(x)=C_{G}(u)$, and let $\log _{s}$ be such that $\log _{s}(u)=x$. This defines an isomorphism between the open sets of regular unipotent and nilpotent elements. The map $\log _{s}$ is defined over the ring $\mathbb{Z}_{(p)}(p$ is good $)$, and $\log _{s}$ can be extended to a homeomorphism $\mathcal{U} \rightarrow \mathcal{N}$. It is now known that $\log _{s}$ is an isomorphism of $G$-varieties for good characteristics since $\mathcal{N}$ is normal [Hum4, p. 118]. However, the map $\log _{s}$ is not uniquely defined and depends on the choice of a regular unipotent element $u$ in $U$ and a regular nilpotent element $x \in \mathfrak{n}$ such that $C_{G}(u)=C_{G}(x)$. For $J \subset \Pi$, let $P_{J}$ be the standard 
parabolic subgroup of $G$ containing the Borel subgroup $B$, and let $P_{J}=L_{J} U_{J}$ with $L_{J}$ being a Levi subgroup and $U_{J}$ the unipotent radical of $P_{J}$. Note that under the adjoint action of $G$, we have $\operatorname{Stab}_{G}\left(U_{J}\right)=P_{J}$ and $\operatorname{Stab}_{G}\left(\mathfrak{n}_{J}\right)=P_{J}$. Also, we have $\log _{s}(U)=\mathfrak{n}$. Let $\mathcal{O}_{J}^{u}$ (resp. $\mathcal{O}_{J}^{n}$ ) be the unipotent (resp. nilpotent) Richardson orbit corresponding to $P_{J}$. We recall that $\mathcal{O}_{J}^{u}$ (resp. $\mathcal{O}_{J}^{n}$ ) is the unique $G$-orbit such that $U_{J} \cap \mathcal{O}_{J}^{u}$ (resp. $\mathfrak{n}_{J} \cap \mathcal{O}_{J}^{n}$ ) is a single open dense $P_{J}$-orbit in $U_{J}$ (resp. $\mathfrak{n}_{J}$ ).

Proposition 1. $\log _{s}\left(U_{J}\right)=\mathfrak{n}_{J}$ and $\log _{s}\left(\mathcal{O}_{J}^{u}\right)=\mathcal{O}_{J}^{n}$.

Proof. Let $U_{L}=U \cap L_{J}$ be the unipotent radical of a Borel subgroup of $L_{J}$ and $\mathfrak{n}_{L}=\operatorname{Lie}\left(U_{L}\right)$. Let $\phi: P_{J} \rightarrow L_{J}$ be the quotient morphism, and let $d \phi: \operatorname{Lie}\left(P_{J}\right) \rightarrow$ $\operatorname{Lie}\left(L_{J}\right)$ be the differential of $\phi$. Under the adjoint action, both $\phi$ and $d \phi$ are $P_{J}$-equivariant with $P_{J}$ acting on $L_{J}$ and $\operatorname{Lie}\left(L_{J}\right)$ by way of the quotient map $P_{J} / U_{J}=L_{J}$. From the definition, we have that $\phi(U)=U_{L}$ and $d \phi(\mathfrak{n})=\mathfrak{n}_{J}$. Let $Y=\log _{s}\left(U_{J}\right)$. Then $Y \subseteq \mathfrak{n}$ is a closed $P_{J}$-stable irreducible subvariety. Let $X=d \phi(Y)$. Then $X$ is an irreducible $P_{J}$-stable subvariety and thus is the closure of a nilpotent $L_{J}$-orbit in $\operatorname{Lie}\left(L_{J}\right)$. Since $L_{J}$ is reductive, then $\operatorname{dim} X=2 \operatorname{dim}\left(X \cap \mathfrak{n}_{L}\right)$. However, $X \subseteq d \phi(\mathfrak{n})=\mathfrak{n}_{L}$, and we have $X=X \cap \mathfrak{n}_{L}$ and $\operatorname{dim} X=\operatorname{dim}\left(X \cap \mathfrak{n}_{L}\right)$. This forces that $\operatorname{dim} X=0$ and $X=\{0\}$, (i.e., the only zero-dimensional nilpotent orbit). Hence, $Y \subset \operatorname{ker}(d \phi)=\mathfrak{n}_{J}$. The map $\log _{s}: U_{J} \rightarrow \mathfrak{n}_{J}$ is a $P_{J}$-equivariant isomorphism and thus sends the unique open $P_{J}$-orbit to the unique open $P_{J}$-orbit. In particular $\log _{s}\left(\mathcal{O}_{J}^{u}\right)=\mathcal{O}_{J}^{n}$.

One of the referees pointed out a different way, using [McN2, Rem. 10], to prove Proposition 1. In fact this argument will prove that for any $G$-equivariant isomorphism $\phi: \mathcal{U} \rightarrow \mathcal{N}$, for any parabolic (in particular Borel) subgroup $P$, Proposition 1 holds with $\log _{s}$ replaced by $\phi$ by using the description of parabolic subgroups given in $[\mathrm{Sp} 2,8.4 .5,8.4 .6(5)]$. The Springer isomorphism assumes that $\phi(U)=\mathfrak{n}$.

The unipotent classes in $G$ and the nilpotent orbits in $\mathfrak{g}$ in characteristic zero are labeled in terms of weighted Dynkin diagrams (cf. [C, Ch. 5, Ch. 13]). This classification still works in good characteristics. For classical groups, the weighted Dynkin diagrams are determined by Jordan types of the unipotent (nilpotent) elements. For exceptional groups, they are listed in the tables in [C, Ch. 13].

Theorem 1. Let $G$ be a connected simple group and $p$ be good for $G$. If $\phi: \mathcal{U} \rightarrow \mathcal{N}$ is a $G$-equivariant isomorphism, then

(a) $\phi$ is a Springer isomorphism.

(b) The one-to-one correspondence defined by $\phi$ between the set of all unipotent conjugacy classes and the set of all nilpotent $G$-orbits preserves the labeling of unipotent classes and nilpotent orbits in terms of weighted-Dynkin diagrams as in $[\mathrm{C}]$ and, in particular, is independent of $\phi$.

(c) For any $r \geq 1$ and $u \in \mathcal{U}, u^{p^{r}}=1$ if and only if $(\phi(u))^{[p]^{r}}=0$.

(d) For each $r \geq 1$, the closed subvarieties $\mathcal{U}_{r}=\left\{u \in \mathcal{U} \mid u^{p^{r}}=1\right\}$ and $\mathcal{N}_{r}=\left\{x \in \mathcal{N} \mid x^{[p]^{r}}=0\right\}$ are isomorphic under $\phi$ and are irreducible.

Proof. (a) By Proposition 1 or [McN2, Rem. 10], $\phi$ in particular sends a regular unipotent element to a regular nilpotent element such that $\phi(U)=\mathfrak{n}$ for any Borel subgroup B. Thus $\phi$ is a Springer isomorphism. 
(b) The independence of $\phi$ follows from (a) and a result of Serre (Appendix to $[\mathrm{McN} 2])$. The correspondence is independent of the choice of Springer isomorphism.

First, by the proposition above, any isomorphism $\phi$ sends a Richardson unipotent class to a Richardson nilpotent orbit of the same type. Since the correspondence of orbits preserves the closure relation, the dimension of the orbit, and the stabilizer subgroup of $G$, it also preserves the component group and the Cartan type of each orbit. In particular, any isomorphism $\phi$ has to send regular unipotent elements to regular nilpotent elements (also note that regular orbits are Richardson orbits).

For classical groups, Springer has constructed explicit isomorphisms. In cases of type $A_{l}$, the map $u \mapsto u-1$ is a $G$-equivariant isomorphism. Note that the unipotent matrix $u$ and the nilpotent matrix $u-1$ give rise to the same partition and the same corresponding weighted Dynkin diagram. This also follows from the fact that all nilpotent orbits (unipotent classes) are Richardson. For symplectic and orthogonal groups, an isomorphism is given by the Cayley transformation $u \mapsto$ $(1-u)(1+u)^{-1}(p \neq 2)$. By expressing the matrix $u$ in its Jordan form, we can easily see that the nilpotent element $(1-u)(1+u)^{-1}$ is associated to the same partition. The weighted Dynkin diagrams are uniquely determined by the partition corresponding to the nilpotent (unipotent) element unless the group is $G=S O_{2 l}$ and the partition consists of all even parts. In this exceptional case, each very even partition corresponds to two different orbits. Both orbits are even and are Richardson orbits. Now Proposition 1 implies that the isomorphism will preserve the weighted Dynkin diagrams in the list of unipotent classes and nilpotent orbits.

For exceptional groups, we can check the list of the properties: dimension, the Levi type of the centralizer, the dimension of unipotent radical of the centralizer and the component group of the centralizer. By inspecting all unipotent classes listed in $[\mathrm{C}]$ with the above listed properties, we find that there are only a few cases when two orbits have the identical properties listed above. But in each case, we can find (by inspecting the Hasse diagrams in $[\mathrm{C}]$ ) a Richardson class which contains only one such orbit in its closure. This also shows that the correspondence is independent of the isomorphism without using Serre's result. Therefore, the correspondence preserves the weighted Dynkin diagrams for exceptional groups.

(c) For classical groups, the conclusion follows from (b) and the fact that $u$ and $\log _{s}(u)$ determine the same partition. For exceptional groups we use the labeling of the unipotent classes and nilpotent orbits. Using the Bala-Carter classification, the label gives the Levi factor and the distinguished parabolic subgroup of the derived group of the Levi factor. The order formula for distinguished unipotent elements was proved by Testerman [T2] and the nilpotency class of a distinguished nilpotent element was provided by McNinch [McN1]. Using these results, a direct comparison for each weighted Dynkin diagram will yield the conclusion.

(d) The isomorphism is a direct result of part (c). The irreducibility follows from [CLNP, Thm. 3.10] and [UGA, Thm. 3.2, §4.1].

We remark that there is a more conceptual proof to the theorem without using Serre's result. In fact, one can use the Bala-Carter classification of unipotent and nilpotent orbits in terms of conjugacy classes of the pairs $(L, P)$ where $L$ is a Levi subgroup and $P$ is a distinguished parabolic subgroup of the semisimple subgroup $L^{\prime}=[L, L]$. For each nilpotent (or unipotent) element $x, L=C_{G}(S)$ with $S$ being a maximal torus of $\operatorname{Stab}_{G}(x)$ and $x$ being a nilpotent (unipotent) Richardson element 
of $P$. Thus any $G$-equivariant isomorphism $\phi: \mathcal{U} \rightarrow \mathcal{N}$ would give the same $G$ conjugacy class of the distinguished pair $(L, P)$. Therefore, any isomorphism $\phi$ (not necessarily a Springer isomorphism) will induce the same correspondence between unipotent classes and nilpotent orbits. We also note that part (c) is proved in $[\mathrm{McN} 1]$.

2.4. It is shown in [NPV] that $\mathcal{N}_{1}=G \cdot \mathfrak{n}_{J}$ for a subset $J \subseteq \Pi$ of simple roots corresponding to a parabolic subalgebra $\mathfrak{p}_{J}$ where $\mathfrak{n}_{J}$ is the nil-radical of $\mathfrak{p}_{J}$. Let $P_{J}$ and $U_{J}$ be the corresponding parabolic and unipotent subgroups of $G$ such that $U_{J}=R_{u}\left(P_{J}\right)$. In [CLNP] representatives for the sets $J$ have been determined. Following Testerman [T1], we define, for each subset $J$ of $\Pi$, the function ht ${ }_{J}$ : $\mathbb{Z} \Phi \rightarrow \mathbb{Z}$ by ht ${ }_{J}\left(\sum_{\alpha \in \Pi} a_{\alpha} \alpha\right)=\sum_{\alpha \notin J} a_{\alpha}$. In particular, the unipotent group $U_{J}$ has nilpotency class at most ht ${ }_{J}\left(\alpha_{0}\right)$, where $\alpha_{0}$ is the highest positive root. Let $\mathcal{U}_{1}=\left\{u \in G \mid u^{p}=1\right\}$. Then $\mathcal{U}_{1}$ is a closed subvariety of $\mathcal{U}$.

Theorem 2. Let $G$ be a simple algebraic group and let $p$ be a good prime. There exists $J \subseteq \Pi$ such that $\mathcal{N}_{1}=G \cdot \mathfrak{n}_{J}$ and the following hold.

(a) For any $\alpha \in \Phi^{+}$, ht $_{J}(\alpha)<p$,

(b) the Lie algebra $\mathfrak{n}_{J}$ has nilpotency less than $p$, and

(c) $\log _{s}: \mathcal{U}_{1} \rightarrow \mathcal{N}_{1}$ is a $G$-equivariant isomorphism. In particular $\mathcal{U}_{1}=G \cdot U_{J}$.

Proof. Note that $\left[\mathfrak{g}_{\alpha}, \mathfrak{g}_{\beta}\right] \subseteq \mathfrak{g}_{\alpha+\beta}$ for all $\alpha, \beta \in \Phi^{+}$, so (b) follows from (a). To prove (a) we use the description of $J$ given in [CLNP]. If $p \geq h$, then (a) is true because all roots $\alpha$ have height at most $h-1$. Assume that $p<h$. For type $A_{l}$, all roots in $\Phi^{+}$have coefficients at most 1 and $|\Pi \backslash J|=p-1$ [CLNP, Thm 3.3]. For type $B_{l}, C_{l}$, we use [CLNP, Thm. 3.8] to see that $|\Pi \backslash J| \leq \frac{p-1}{2}$ and the fact that all roots in $\Phi^{+}$have coefficients at most 2 . For type $D_{l}$, we have $|\Pi \backslash J| \leq \frac{p+1}{2}$ if $J$ contains both $\alpha_{l-1}$ and $\alpha_{l}$ and $|\Pi \backslash J| \leq \frac{p-1}{2}$ otherwise. Now (a) follows from the fact that the coefficients of $\alpha_{l-1}$ and $\alpha_{l}$ are at most 1 in the case of type $D_{l}$.

For the exceptional groups, the sets $J$ are determined in [CLNP, 4.4]. For the reader's convenience, we list them here together with $\alpha_{0}$. We can read off ht ${ }_{J}\left(\alpha_{0}\right)$ from the tables as the sum of the coefficients in $\alpha_{0}$ of simple roots not in $J$. In each case, we have ht ${ }_{J}\left(\alpha_{0}\right)<p$.

Type $E_{6}: \alpha_{0}=\alpha_{1}+2 \alpha_{2}+2 \alpha_{3}+3 \alpha_{4}+2 \alpha_{5}+\alpha_{6}$.

\begin{tabular}{|c|c|c|}
\hline$p$ & $J$ & $\mathrm{ht}_{J}\left(\alpha_{0}\right)$ \\
\hline 5 & $\{1,2,4,6\}$ & 4 \\
\hline 7 & $\{2,3,5\}$ & 5 \\
\hline 11 & $\{4\}$ & 8 \\
\hline
\end{tabular}

Type $E_{7}: \alpha_{0}=2 \alpha_{1}+2 \alpha_{2}+3 \alpha_{3}+4 \alpha_{4}+3 \alpha_{5}+2 \alpha_{6}+\alpha_{7}$.

\begin{tabular}{|c|c|c|}
\hline$p$ & $J$ & $\mathrm{ht}_{J}\left(\alpha_{0}\right)$ \\
\hline 5 & $\{1,2,3,5,6,7\}$ & 4 \\
\hline 7 & $\{1,2,3,5,7\}$ & 6 \\
\hline 11 & $\{2,3,5\}$ & 9 \\
\hline 13 & $\{4,6\}$ & 11 \\
\hline 17 & $\{4\}$ & 13 \\
\hline
\end{tabular}


Type $E_{8}: \alpha_{0}=2 \alpha_{1}+3 \alpha_{2}+4 \alpha_{3}+6 \alpha_{4}+5 \alpha_{5}+4 \alpha_{6}+3 \alpha_{7}+2 \alpha_{8}$.

\begin{tabular}{|c|c|c|}
\hline$p$ & $J$ & ht $_{J}\left(\alpha_{0}\right)$ \\
\hline 7 & $\{1,2,3,5,6,7,8\}$ & 6 \\
\hline 11 & $\{1,2,3,5,6,8\}$ & 7 \\
\hline 13 & $\{2,3,5,6,8\}$ & 9 \\
\hline 17 & $\{2,3,5,7\}$ & 14 \\
\hline 19 & $\{2,3,5\}$ & 17 \\
\hline 23 & $\{4,6\}$ & 19 \\
\hline 29 & $\{4\}$ & 23 \\
\hline
\end{tabular}

Type $F_{4}: \alpha_{0}=2 a f_{1}+3 \alpha_{2}+4 \alpha_{3}+2 \alpha_{4}$.

\begin{tabular}{|c|c|c|}
\hline$p$ & $J$ & ht $_{J}\left(\alpha_{0}\right)$ \\
\hline 5 & $\{1,3,4\}$ & 3 \\
\hline 7 & $\{1,3\}$ & 5 \\
\hline 11 & $\{3\}$ & 8 \\
\hline
\end{tabular}

Type $G_{2}: \alpha_{0}=3 \alpha_{1}+2 \alpha_{2}$.

\begin{tabular}{|c|c|c|}
\hline$p$ & $J$ & ht $_{J}\left(\alpha_{0}\right)$ \\
\hline 5 & $\{2\}$ & 3 \\
\hline
\end{tabular}

For part (c), the fact that $\log _{s}$ restricts to a $G$-equivariant isomorphism follows from Theorem 1 , and the statement that $\mathcal{U}_{1}=G \cdot U_{J}$ is a consequence of Proposition 1.

We remark that although $\log _{s}$ is a $G$-equivariant isomorphism between $\mathcal{U}_{1}$ and $\mathcal{N}_{1}$, in general, $\log _{s}$ does not preserve the group structure when restricted to unipotent abelian subgroups. For example, the Springer map used in the proof of Theorem 1 is not a group homomorphism in general. For the remainder of this section we investigate the exponential maps, exp, which are only defined over nilpotent Lie subalgebras of nilpotency less than $p$ [Sei]. The advantage of the exponential map is that it is a group homomorphism when restricted to certain unipotent subgroups.

2.5. The subgroup $U_{J}$ of $G$ has a $\mathbb{Z}$-structure with the $\mathbb{Z}$-form $\left(U_{J}\right)_{\mathbb{Z}}$. The Lie algebra $\mathfrak{n}_{J}$ also has a $\mathbb{Z}$-structure $\left(\mathfrak{n}_{J}\right)_{\mathbb{Z}}$ as a scheme. Because the nilpotency class of $\mathfrak{n}_{J}$ is less than $p$, by Seitz [Sei, Prop. 5.1-5.3] (with $U_{J}=U_{\Gamma}$ and $\Gamma=\Phi^{+} \backslash \Phi_{J}^{+}$, in the notation of [Sei, Prop. 5.1]) there is an isomorphism of algebraic $k$-varieties $\exp : \mathfrak{n}_{J} \rightarrow U_{J}$, which is defined over the localization $\mathbb{Z}_{(p)}$. For $J$ satisfying the conditions of Theorem 2 , we can check case by case that, for all $x \in \mathfrak{n}_{J}\left(\mathbb{Z}_{(p)}\right)$, the conditions of $[\mathrm{T} 2$, Lem. 1.4] are satisfied, except in a few cases for which we cannot find $J_{1}$ and $J_{2}$. But in those cases, one of the $e_{1}$ and $e_{2}$ can be chosen as a root vector, and the proof of [T2, Lem. 1.4] still works. When $G$ is of adjoint type, the map exp was established in [T2, Lem. 1.1] for all $x \in \mathfrak{n}_{J}\left(\mathbb{Z}_{(p)}\right)$. The condition required for [T2, Lem. 1.1] is guaranteed in [T2, Lem. 1.4]. For simply connected groups, the map exp is first defined over the field of complex numbers via the usual exponential map. Seitz [Sei, Prop 5.1] proved that the exponential map is actually defined over $\mathbb{Z}_{(p)}$. In particular, given any $x \in \mathfrak{n}_{J}\left(\mathbb{Z}_{(p)}\right)$ and any rational $G_{\mathbb{Z}_{(p)}}{ }^{-}$ module $M\left(\mathbb{Z}_{(p)}\right)$, the action of $\exp (x)$ on $M\left(\mathbb{Z}_{(p)}\right)$ is given by $\exp (x)=\sum_{n=0}^{\infty} x^{n} / n$ ! with $x^{n} / n$ ! being elements of the distribution algebra of $\left(U_{J}\right)_{\mathbb{Z}_{(p)}}$ (see [Jan1, I, Ch. $7])$. For any $x \in \mathfrak{n}_{J}\left(\mathbb{F}_{p}\right)$, we still use $x^{(n)}$ to denote the image of $z^{n} / n$ ! in the 
distribution algebra of $\left(U_{J}\right)_{\mathbb{F}_{p}}$ for some $z \in \mathfrak{n}_{J}\left(\mathbb{Z}_{(p)}\right)$ which has image $x$ by reduction modulo $p$. The following proposition indicates how these structures behave under base change.

Proposition 2. Let $x_{1}, x_{2} \in \mathfrak{n}_{J}\left(\mathbb{F}_{p}\right)$ such that $\left[x_{1}, x_{2}\right]=0$. Suppose that $M$ is a rational $G$-module with a $G_{\mathbb{F}_{p}}$-structure, $M_{\mathbb{F}_{p}}$. Then for $t_{1}$ and $t_{2}$ in $k$, the action of $\exp \left(t_{1} x_{1}+t_{2} x_{2}\right)$ on $M=M\left(\mathbb{F}_{p}\right) \otimes_{\mathbb{F}_{p}} k$ is given by the exponential map

$$
\exp \left(t_{1} x_{1}+t_{2} x_{2}\right)=\sum_{n, m=0}^{\infty}\left(x_{1}^{(n)} x_{2}^{(m)}\right) \otimes t_{1}^{n} t_{2}^{m} .
$$

Proof. Note that $\mathfrak{n}_{J}$ is an algebraic group with multiplication given by the Hausdorff formula [Ser, IV. 8] and exp is an isomorphism of algebraic groups $\mathfrak{n}_{J} \rightarrow U_{J}$ by [Sei, Prop. 5.2]. Since $\left[x_{1}, x_{2}\right]=0$, the Hausdorff formula implies $\exp \left(t_{1} x_{1}+t_{2} x_{2}\right)=$ $\exp \left(t_{1} x_{1}\right) \exp \left(t_{2} x_{2}\right)$ in $U_{J}$. Now we see from the definition of the exponential map, exp, that the formula holds in $\operatorname{End}_{k}(M)$ (see [Jan1, I. 7.4(1)]).

2.6. It is well known that $N_{G}\left(\mathfrak{n}_{J}\right)=N_{G}\left(U_{J}\right)=P_{J}$ and that $N_{G}\left(\mathfrak{n}_{J}\right)$ is a connected closed subgroup of $G$ and defined over $\mathbb{Z}_{(p)}$ (see [Jan1, 2.6], for example). The following proposition lists some properties of the exponential map. A subgroup $A$ of $G$ of type $A_{1}$ is called a good $A_{1}$-subgroup if all weights of Lie $(G)$ are at most $2(p-1)$ as an $A$-module. The 1-dimensional unipotent algebraic group $G_{a}$ has Lie algebra $k \gamma^{(1)}$ and its distribution algebra $\operatorname{Dist}\left(G_{a}\right)$ has a $k$-basis $\left\{\gamma^{(i)} \mid i=0,1,2, \ldots\right\}$ which satisfies $\gamma^{(i)} \gamma^{(j)}=\left(\begin{array}{c}i+j \\ i\end{array}\right) \gamma^{(i+j)}$.

Lemma 2. [Sei] Let $p$ be a good prime for the group $G$. For any $1 \neq u \in \mathcal{U}_{1}$, there exists a unique homomorphism $f_{u}: G_{a} \rightarrow G$ such that $f_{u}\left(G_{a}\right)=U_{A}$ is the unipotent subgroup of a Borel subgroup $B_{A}$ of a good $A_{1}$-subgroup $A$ of $G$ with the following properties:

(i) $f_{u}(1)=u$;

(ii) $d f_{u}\left(\gamma^{(1)}\right)=e$ is a basis for $\operatorname{Lie}\left(U_{A}\right)$ and, identifying $\operatorname{Lie}\left(U_{A}\right)$ with $G_{a}$ under the map $e \mapsto 1$, the homomorphism $f_{u}: \operatorname{Lie}\left(U_{a}\right) \rightarrow U$ has differential $d f_{u}=1$ on $\operatorname{Lie}\left(U_{A}\right)$;

(iii) $C_{G}(u)=C_{G}\left(U_{A}\right)=C_{G}\left(\operatorname{Lie}\left(U_{A}\right)\right)$.

Proposition 3. Let $p$ be good and $\mathcal{N}_{1}=G \cdot \mathfrak{n}_{J}$.

(a) [Sei, 5.3] The isomorphism exp $: \mathfrak{n}_{J} \rightarrow U_{J}$ is invariant under the adjoint action of $P_{J}$ and the differential d $\exp : \mathfrak{n}_{J} \rightarrow \mathfrak{n}_{J}$ is the identity map.

(b) [Sei, Lem. 6.3] $C_{P_{J}}(x)=C_{P_{J}}(\exp (x))$ for all $x \in \mathfrak{n}_{J}$.

2.7. One of the consequences of Theorem 2(c) is that for each unipotent element $u \in G$ with $u^{p}=1$, we can assume $u \in U_{A} \subseteq U_{J}$ with $\operatorname{Lie}\left(U_{A}\right)=k e \subseteq \mathfrak{n}_{J}$, where $U_{A}$ is the unipotent subgroup of a Borel subgroup of a good $A_{1}$-subgroup of $G$. By Lemma 2(iii), we have $C_{G}(u)=C_{G}(k e)$.

Let $x \in \mathfrak{n}_{J}\left(\mathbb{F}_{p}\right)$ be an $\mathbb{F}_{p}$-rational point in $\mathfrak{n}_{J}$. The centralizer $C_{G}(x)$ is a closed subgroup of $G$ defined over $\mathbb{F}_{p}[\operatorname{Sp} 2,11.2 .1]$. Thus the map $G \rightarrow G \cdot x$ of algebraic varieties is defined over $\mathbb{F}_{p}$. We want to extend the map exp : $\mathfrak{n}_{J} \rightarrow U_{J}$ to a map $\exp : \mathcal{N}_{1} \rightarrow \mathcal{U}_{1}$. The first step is to compare $C_{G}(x)$ with $C_{G}(\exp (x))$ for $x \in \mathfrak{n}_{J}$.

Proposition 4. Let $u \in U_{A} \subseteq U_{J}$ be an element where $U_{A}$ is the unipotent radical of a Borel subgroup $B_{A}$ of a good $A_{1}$-subgroup of $G$.

(a) If $B_{A} \subseteq P_{J}$, then $C_{G}(x)=C_{G}(\exp (x))$ for each $x \in \operatorname{Lie}\left(U_{A}\right)$. 
(b) For good primes $p$, if $x \in \mathfrak{n}_{J}$ is a Richardson element for $P_{J}$, then $C_{G}(x)=$ $C_{G}(\exp (x))$.

(c) If either $\mathcal{N}_{1}$ or $\mathcal{U}_{1}$ is a normal variety, then for all $x \in \mathcal{N}_{1}$, we have $C_{G}(x)=$ $C_{G}(\exp (x))$.

Proof. (a) Let $f: G_{a} \rightarrow U_{A}$ be an isomorphism of algebraic groups. Since $U_{A} \subseteq U_{J}$, Proposition 5.4 of [Sei] implies that there are $e_{i} \in \mathfrak{n}_{J}(i=0,1,2, . .$,$) such that$ $\left[e_{i}, e_{j}\right]=0$ and $f(t)=\exp \left(t e_{0}\right) \exp \left(t^{p} e_{1}\right) \cdots$. Let $B_{A}=U_{A} T_{A}$ be a Borel subgroup of $A$ where $T_{A} \subseteq P_{J}$ is a maximal torus of $A$. Note each factor of $f(t)$ is invariant under the $T_{A}$-action. Using the ideas in the proof of [Sei, Prop. 5.5], we show that $e_{i}$ has $T_{A}$-weight $2 p^{i}$. Since $A$ is a good $A_{1}, e_{i}=0$ for all $i \geq 1$ and $f(t)=\exp \left(t e_{0}\right)$ for some $e_{0} \in \mathfrak{n}_{J}$. Moreover, $d \exp$ is the identity on $\mathfrak{n}_{J}$ by [Sei, Prop. 5.3]. Thus, $e_{0} \in \operatorname{Lie}\left(U_{A}\right)$ and $\exp \left(e_{0}\right)=u$. Now (a) follows from [Sei, Prop. 6.1] (cf. Lemma 2(iii)).

(b) Let $x \in \mathfrak{n}_{J}$ be a Richardson element of $P_{J}$. Because $\exp _{x}: G_{a} \rightarrow U_{J}$ defined by $\exp _{x}(t)=\exp (t x)$ is a homomorphism of algebraic groups whose image $\exp _{x}(k) \subseteq U_{J}$ is a closed subgroup and because Lie $\left(\exp _{x}(k)\right)=k x$, we have $C_{G}(x) \supseteq C_{G}(\exp (x))$. Let $x_{1}=\log _{s}(\exp (x))$, where $\log _{s}$ is a Springer isomorphism. By Proposition 1, we have $x_{1} \in \mathfrak{n}_{J}$ is a Richardson element. Thus there exists $g_{0} \in P_{J}$ such that $x=g_{0} \cdot x_{1}$. Since $\log _{s}$ is a $G$-equivariant morphism, we have that

$$
C_{G}(\exp (x))=C_{G}\left(\log _{s}(\exp (x))\right)=C_{G}\left(x_{1}\right)=g_{0}^{-1} C_{G}(x) g_{0} .
$$

Using the inclusion $C_{G}(\exp (x)) \subseteq C_{G}(x)$, we see that $g_{0} \in N_{G}\left(C_{G}(x)\right)$ and $C_{G}(x)=$ $C_{G}(\exp (x))$.

(c) From part (b), $\exp : G \cdot x \rightarrow G \cdot \exp (x)$ is a $G$-equivariant isomorphism (since its differential is not zero). Under the Springer isomorphism, both $G \cdot \mathfrak{n}_{J}$ and $G \cdot U_{J}$ are normal algebraic varieties. Since the complement of the dense $G$-orbit in $G \cdot \mathfrak{n}_{J}$ has codimension at least $2[\operatorname{Jan} 4,8.4]$, by (b) the isomorphism on the Richardson orbit induced by exp extends uniquely to an isomorphism exp : $\mathcal{N}_{1} \rightarrow \mathcal{U}_{1}$. The map exp : $\mathcal{N}_{1} \rightarrow \mathcal{U}_{1}$ is $G$-equivariant since its restriction to the open orbit is $G$ equivariant.

We expect that condition (a) holds for every $U_{A} \subseteq U_{J}$. This condition is equivalent to the fact that $x$ has an associated character (see [Jan4, 5.3]) with image in $P_{J}$. In many cases we know that $\mathcal{N}_{1}$ is normal. For groups of type $A$, Donkin [D] showed that all orbit closures are normal. Furthermore, the closures of the regular and subregular orbits [KLT] are normal. Thomsen [Th] has also provided a method to show that $\mathcal{N}_{1}$ is normal for several cases for exceptional groups. It is also an interesting question to know whether exp $: \mathfrak{n}_{J} \rightarrow U_{J}$ is the restriction of a Springer isomorphism. If this is the case, the assumption of the normality of $\mathcal{N}_{1}$ can be dropped.

2.8. For any $x \in \mathfrak{n}_{J}\left(\mathbb{F}_{p}\right)$, we define $\psi_{x}: G \rightarrow \mathcal{N}_{1}$ by $\psi_{x}(g)=g \cdot x$. The morphism $\psi_{x}$ is defined over $\mathbb{F}_{p}$. We define $V_{x}=\left\{g \in G \mid g \cdot x \in \mathfrak{n}_{J}\right\}$. Then $V_{x}=\psi_{x}^{-1}\left(\mathfrak{n}_{J}\right)$ is a closed subvariety defined over $\mathbb{F}_{p}$ by Lemma 1 . For any $g \in V_{x}$ set $\phi_{x}(g)=$ $\exp (g \cdot x) g \exp (x)^{-1} g^{-1}$. The map $\phi_{x}: V_{x} \rightarrow G$ is a morphism of algebraic varieties over $k$.

Lemma 3. For $x \in \mathfrak{n}_{J}\left(\mathbb{F}_{p}\right)$, the map $\phi_{x}$ is defined over $\mathbb{F}_{p}$. That is, there exists an $\mathbb{F}_{p}$-morphism $\left(\phi_{x}\right)_{\mathbb{F}_{p}}:\left(V_{x}\right)_{\mathbb{F}_{p}} \rightarrow G_{\mathbb{F}_{p}}$ such that $\phi_{x}=\left(\phi_{x}\right)_{\mathbb{F}_{p}} \otimes_{\mathbb{F}_{p}} k$. 
Proof. We first recall that $\mathcal{N}$ has an $\mathbb{F}_{p}$-structure and the adjoint action $G \times \mathcal{N} \rightarrow \mathcal{N}$ is defined over $\mathbb{F}_{p}$. The restricted nullcone $\mathcal{N}_{1}$ is a subvariety of $\mathcal{N}$ with $\mathbb{F}_{p}$-structure since the $p$-map $[p]$ is a morphism of algebraic varieties and defined over $\mathbb{F}_{p}$. Let $f_{\mathbb{F}_{p}}$ be the composition of $\left(V_{x}\right)_{\mathbb{F}_{p}} \stackrel{\psi_{x}}{\rightarrow}\left(\mathfrak{n}_{J}\right)_{\mathbb{F}_{p}} \stackrel{\exp }{\rightarrow}\left(U_{J}\right)_{\mathbb{F}_{p}}$ such that $g \mapsto g \cdot x \mapsto \exp (g \cdot x)$. Let $\iota_{\mathbb{F}_{p}}, h_{\mathbb{F}_{p}}: G_{\mathbb{F}_{p}} \rightarrow G_{\mathbb{F}_{p}}$ be the morphisms of varieties defined by $\iota_{\mathbb{F}_{p}}(g)=g^{-1}$ and $h_{\mathbb{F}_{p}}(g)=g \exp (x)^{-1}$ for all $g \in G$. Let $m_{\mathbb{F}_{p}}: G_{\mathbb{F}_{p}} \times G_{\mathbb{F}_{p}} \times G_{\mathbb{F}_{p}} \rightarrow G_{\mathbb{F}_{p}}$ and $\Delta_{\mathbb{F}_{p}}: G_{\mathbb{F}_{p}} \rightarrow G_{\mathbb{F}_{p}} \times G_{\mathbb{F}_{p}} \times G_{\mathbb{F}_{p}}$ be the product and diagonal morphisms respectively. Then the composition

$$
\phi_{x, \mathbb{F}_{p}}=m_{\mathbb{F}_{p}} \circ\left(f_{\mathbb{F}_{p}} \times h_{\mathbb{F}_{p}} \times \iota_{\mathbb{F}_{p}}\right) \circ \Delta_{\mathbb{F}_{p}}
$$

defines an $\mathbb{F}_{p}$-morphism $\left(V_{x}\right)_{\mathbb{F}_{p}} \rightarrow G_{\mathbb{F}_{p}}$ such that $\left(\phi_{x}\right)_{\mathbb{F}_{p}} \otimes_{\mathbb{F}_{p}} k=\phi_{x}$.

Proposition 5. Let $G$ be a simple algebraic group and suppose that $p$ is good for $G$. Assume that $\mathcal{N}_{1}$ is normal.

(a) For any $x \in \mathfrak{n}_{J}\left(\mathbb{F}_{p}\right), \phi_{x}: V_{x} \rightarrow G$ is a constant morphism of algebraic varieties.

(b) For any $x \in \mathfrak{n}_{J}\left(\mathbb{F}_{p}\right)$, the map $\exp : G \cdot x \rightarrow G$ defined by $g \cdot x \mapsto g \exp (x) g^{-1}$ is a $G$-equivariant morphism extending the morphism $\exp :(G \cdot x) \cap \mathfrak{n}_{J} \rightarrow G$.

(c) The map $\exp : G \cdot x \rightarrow G \cdot \exp (x)$ is an isomorphism of $G$-orbits for every $x \in \mathfrak{n}_{J}$.

Proof. (a) The morphism $\left(\phi_{x}\right)_{\mathbb{F}_{p}}$ is actually defined over the local ring $\mathbb{Z}_{(p)}$ from the construction of exp in Section 2. It is known that over $\mathbb{Z}_{(p)}$, the corresponding morphism is constant. Thus $\phi_{x}$ is also a constant morphism (see Lemma 1).

(b) First consider the map $f_{x}: G \rightarrow G$ by $f_{x}(g)=g \exp (x) g^{-1}$. By Proposition 4(c), we have $\operatorname{Stab}_{G}(x)=C_{G}(\exp (x))$ and $f_{x}$ factors through $G \rightarrow G \cdot x \cong$ $G / \operatorname{Stab}_{G}(x)$ to get a morphism $\bar{f}_{x}: G \cdot x \rightarrow G$ by the universal property of the quotient varieties by a group action. We still need to show that $\bar{f}_{x}:(G \cdot x) \cap \mathfrak{n}_{J} \rightarrow G$ is the same as exp when restricted to $G \cdot x \cap \mathfrak{n}_{J}$. If $x^{\prime} \in \mathfrak{n}_{J} \cap G \cdot x$, then there exists $g \in V_{x}$ such that $g \cdot x=x^{\prime} \in \mathfrak{n}_{J}$. By (a), we have $\phi_{x}(g)=1$ in $G$, i.e.,

$$
\bar{f}_{x}\left(x^{\prime}\right)=g \exp (x) g^{-1}=\exp (g \cdot x)=\exp \left(x^{\prime}\right) .
$$

Part (c) follows from Proposition 4(c).

2.9. We can now prove the main result of this section concerning the exponential map over the restricted nullcone.

Theorem 3. Let $G$ be a simple algebraic group such that $G_{s c} \rightarrow G$ is separable and let $p$ be a good prime for $G$. Assume that $\mathcal{N}_{1}$ is normal. With $J$ defined in Theorem 2 such that $\mathcal{N}_{1}=G \cdot \mathfrak{n}_{J}$, then the isomorphism exp : $\mathfrak{n}_{J} \rightarrow U_{J}$ extends uniquely to a $G$-equivariant isomorphism $\exp : \mathcal{N}_{1} \rightarrow \mathcal{U}_{1}$ of algebraic varieties and has the following properties:

(a) For any $0 \neq x \in \mathcal{N}_{1}$, the map exp restricted to $k x$ is a homomorphism of algebraic groups $\exp : k x \rightarrow U$ that can be extended to a good $A_{1}$.

(b) For any $x, y \in \mathcal{N}_{1},[x, y]=0$ if and only if $[\exp (x), \exp (y)]=1$.

(c) The isomorphism is defined over $\mathbb{F}_{p}$.

For the rest of the paper, we call exp the exponential map. The inverse map $\log : \mathcal{U}_{1} \rightarrow \mathcal{N}_{1}$ is the logarithmic map. 
Proof. First the isomorphism exp : $\mathfrak{n}_{J} \rightarrow \mathcal{U}_{J}$ extending uniquely to exp : $\mathcal{N}_{1} \rightarrow \mathcal{U}_{1}$ was proved in Proposition 4.

To prove (a), we can assume that $0 \neq x \in \mathfrak{n}_{J}$. The map $\exp _{x}: G_{a} \rightarrow U_{J}$ $(t \mapsto \exp (t x))$ defines a homomorphism of algebraic groups which can be extended to a good $A_{1}$ using the argument of [Sei, Prop. 5.5]. We now use the argument of [Sei, Lem. 6.3] to see that $y \in C_{\mathfrak{g}}(x)$ if and only if $y \in C_{\mathfrak{g}}(\exp (x))$. For the same reason, we can assume that $y \in \mathfrak{n}_{J}$ and then, by [Sei, Prop. 6.1], we have $\exp (x) \in C_{G}(y)=C_{G}(\exp (y))$. Thus (b) follows.

For (c), the map exp : $\mathfrak{n}_{J} \rightarrow \mathcal{U}_{J}$ is defined over $\mathbb{F}_{p}$. Thus its extension is also defined over $\mathbb{F}_{p}$ since both $\mathcal{N}_{1}$ and $\mathcal{U}_{1}$ are defined over $\mathbb{F}_{p}$.

\section{3. $\mathbb{F}_{p}$-EXPRESSIBILITY}

3.1. In this section we assume that the prime $p$ is good for the simple algebraic group $G$ and that $\mathcal{N}_{1}$ is normal. Let exp : $\mathcal{N}_{1} \rightarrow \mathcal{U}_{1}$ be the exponential map and let $\log : \mathcal{U}_{1} \rightarrow \mathcal{N}_{1}$ be the logarithmic map.

Definition 1. An element $x \in \mathfrak{g}$ is $\mathbb{F}_{p}$-expressible if $x=\sum_{i=1}^{n} c_{i} x_{i}$ where $c_{i} \in k$, $x_{i} \in \mathfrak{g}\left(\mathbb{F}_{p}\right), x_{i}^{[p]}=0$, and $\left[x_{i}, x_{j}\right]=0$ for $1 \leq i, j \leq n$.

Let $\mathcal{N}^{\mathbb{F}_{p}}$ be the set of $\mathbb{F}_{p}$-expressible elements of $\mathfrak{g}$. From the definition given above we have $\mathcal{N}^{\mathbb{F}_{p}} \subseteq \mathcal{N}_{1}$. The adjoint action of the algebraic group $G$ on the variety $\mathcal{N}_{1}$ induces an action of $G\left(\mathbb{F}_{p}\right)$ on the variety $\mathcal{N}^{\mathbb{F}_{p}}$. $G$.

We can also formulate an analogous notion of $\mathbb{F}_{p}$-expressibility for elements in

Definition 2. An element $g \in G$ is $\mathbb{F}_{p}$-expressible if

$$
g=\exp \left(t_{1} x_{1}\right) \exp \left(t_{2} x_{2}\right) \ldots \exp \left(t_{n} x_{n}\right)
$$

where $t_{i} \in k, x_{i} \in \mathfrak{g}\left(\mathbb{F}_{p}\right), x_{i}^{[p]}=0$, and $\left[x_{i}, x_{j}\right]=0$ for $1 \leq i, j \leq n$.

Let $\mathcal{U}^{\mathbb{F}_{p}}$ be the variety of $\mathbb{F}_{p}$-expressible elements of $G$. If $g \in \mathcal{U}^{\mathbb{F}_{p}}$, then $g^{p}=1$, and so $\mathcal{U}^{\mathbb{F}_{p}} \subseteq \mathcal{U}$. Similarly, $G\left(\mathbb{F}_{p}\right)$ acts on $\mathcal{U}^{\mathbb{F}_{p}}$.

3.2. Let $\mathcal{N}_{[2]}=\left\{(x, y) \in \mathcal{N}_{1} \times \mathcal{N}_{1}:[x, y]=0\right\}$. This is the restricted variety of commuting nilpotent elements in $\mathfrak{g}$. $G$ acts on $\mathcal{N}_{[2]}$ via diagonal adjoint action and $\mathcal{N}_{[2]}$ is defined over $\mathbb{F}_{p}$. Let us formulate the following assumption on $\mathfrak{g}$.

Assumption 1. Let $J \subset \Delta$ be given as in Theorem 2 with $\mathcal{N}_{1}=G \cdot \mathfrak{n}_{J}$. Assume that $\mathcal{N}_{[2]}=G \cdot\left(\left(\mathfrak{n}_{J} \times \mathfrak{n}_{J}\right) \cap \mathcal{N}_{[2]}\right)$.

For $p \geq h$, we have $\mathfrak{n}=\mathfrak{n}_{J}$ and the assumption is true, because $x, y$ generates a nilpotent Lie subalgebra, which is conjugated under $G$ to an abelian subalgebra of $\mathfrak{n}$. It is interesting to note that in order to verify the assumption for smaller primes we may need new information about the computation for support varieties for the second Frobenius kernel of $G$. The support variety of the trivial module for $G_{2}$ in that case is $\mathcal{N}_{[2]}$.

We also note that $G \cdot\left(\left(\mathfrak{n}_{J} \times \mathfrak{n}_{J}\right) \cap \mathcal{N}_{[2]}\right)$ is always a closed subvariety of $\mathcal{N}_{[2]}$. In fact,

$$
Z=\left\{\left(g P_{J}, z\right) \in G / P_{J} \times \mathcal{N}_{[2]} \mid g \cdot z \in \mathfrak{n}_{J} \times \mathfrak{n}_{J}\right\}
$$

is a closed subvariety of $G / P_{J} \times \mathcal{N}_{[2]}$ and $G \cdot\left(\mathfrak{n}_{J} \times \mathfrak{n}_{J}\right) \cap \mathcal{N}_{[2]}$ is the image of $Z$ under the projection to the second component. Because $G / P_{J}$ is a complete algebraic variety, it follows that $G \cdot\left(\mathfrak{n}_{J} \times \mathfrak{n}_{J}\right) \cap \mathcal{N}_{[2]}$ is closed in $\mathcal{N}_{[2]}$. 
3.3. The two notions of $\mathbb{F}_{p}$-expressibility are related using the exponential map. This is shown in the following result.

Proposition 6. Suppose that Assumption 1 is satisfied. Let $x_{1}, x_{2}, \ldots, x_{n} \in \mathcal{N}_{1}$ such that $\left[x_{i}, x_{j}\right]=0$ for all $i, j=1,2, \ldots, n$.

(a) For any $t_{1}, t_{2}, \ldots, t_{n} \in k$,

$$
\exp \left(\sum_{i=1}^{n} t_{i} x_{i}\right)=\prod_{i=1}^{n} \exp \left(t_{i} x_{i}\right)
$$

(b) An element $g$ of $G$ is $\mathbb{F}_{p}$-expressible if and only if $g=\exp (x)$ for some $x \in \mathcal{N}^{\mathbb{F}_{p}}$. Moreover, the map exp maps $\mathcal{N}^{\mathbb{F}_{p}}$ onto $\mathcal{U}^{\mathbb{F}_{p}}$. In particular, for any element $1 \neq g \in G\left(\mathbb{F}_{p}\right)$ such that $g^{p}=1$, then $g \in \mathcal{U}_{1}^{\mathbb{F}_{p}}$.

Proof. (a) Since $\mathcal{N}_{1}$ is conical, we can use induction on $n$ to reduce to proving $\exp (x+y)=\exp (x) \exp (y)$ for all $x, y \in \mathcal{N}_{1}$ such that $[x, y]=0$. If there is $g \in G$ such that $g \cdot x$ and $g \cdot y$ are both in $\mathfrak{n}_{J}$, then we are done.

Define a map $f: \mathcal{N}_{[2]} \rightarrow G$ by

$$
f(x, y)=\exp (x+y) \exp (-x) \exp (-y), \quad \text { for all }(x, y) \in \mathcal{N}_{[2]} \text {. }
$$

Clearly $f$ is a morphism of algebraic varieties and $G$-equivariant. By Proposition 2, $f\left(\mathcal{N}_{[2]} \cap\left(\mathfrak{n}_{J} \times \mathfrak{n}_{J}\right)\right)=1$, where 1 is the identity element of the group $G$. Thus $f\left(G \cdot\left(\mathcal{N}_{[2]} \cap\left(\mathfrak{n}_{J} \times \mathfrak{n}_{J}\right)\right)\right)=\{1\}$. By the assumption, we have $f\left(\mathcal{N}_{[2]}\right)=\{1\}$ and $\exp (x+y)=\exp (x) \exp (y)$ for all $x, y \in \mathcal{N}_{1}$ with $[x, y]=0$. Part (b) is a direct consequence of part (a) since exp is defined over $\mathbb{F}_{p}$.

3.4. We can now show that the exponential map sends $\mathbb{F}_{p}$-expressible elements in $\mathfrak{g}$ to $\mathbb{F}_{p}$-expressible elements in $G$.

Theorem 4. The $G$-equivariant isomorphism exp : $\mathcal{N}_{1} \rightarrow \mathcal{U}_{1}$ induces a $G\left(\mathbb{F}_{p}\right)$ equivariant isomorphism $\exp : \mathcal{N}^{\mathbb{F}_{p}} \rightarrow \mathcal{U}^{\mathbb{F}_{p}}$ of varieties.

Proof. Let $x=\sum_{i=1}^{n} c_{i} x_{i} \in \mathcal{N}^{\mathbb{F}_{p}}$. By Proposition 6 , we then have $\exp (x)=$ $\prod_{i=1}^{n} \exp \left(c_{i} x_{i}\right)$. Thus $\exp \left(\mathcal{N}^{\mathbb{F}_{p}}\right) \subseteq \mathcal{U}^{\mathbb{F}_{p}}$. Conversely, any

$$
g=\exp \left(t_{1} x_{1}\right) \exp \left(t_{2} x_{2}\right) \cdots \exp \left(t_{n} x_{n}\right)=\exp \left(\sum_{i=1}^{n} t_{i} x_{i}\right) \in \exp \left(\mathcal{N}^{\mathbb{F}_{p}}\right) .
$$

\section{Finite MORPhisms OF SUPPORT VARIETIES}

Unless otherwise stated, we will assume throughout this section that $p$ is good, $\mathcal{N}_{1}$ is normal, and Assumption 1 is satisfied. In particular when $p \geq h$, these conditions are automatically satisfied.

4.1. For each $x \in \mathcal{N}_{1}$, we denote by $\hat{x}: G_{a} \rightarrow G$ the homomorphism of algebraic groups defined by $\hat{x}(t)=\exp (t x)$ (see Theorem 3 ).

Let $E$ be an elementary abelian $p$-subgroup of $G\left(\mathbb{F}_{p}\right)$ of rank $n$. We may write $E=\left\langle y_{1}\right\rangle \times\left\langle y_{2}\right\rangle \times \cdots \times\left\langle y_{n}\right\rangle$ with independent generators $y_{1}, y_{2}, \ldots, y_{n}$. From Proposition 6(b), there exist nilpotent elements of $\mathcal{N}^{\mathbb{F}_{p}}$, namely $x_{1}, x_{2}, \ldots, x_{n}$ such that $\widehat{x}_{i}(1)=y_{i}$ for $i=1,2, \ldots, n$. We can now define a map $\sigma: \mathbb{A}^{n} \rightarrow G$ of algebraic varieties by $\sigma\left(t_{1}, \ldots, t_{n}\right)=\widehat{x}_{1}\left(t_{1}\right) \widehat{x}_{2}\left(t_{2}\right) \cdots \widehat{x}_{n}\left(t_{n}\right)$. Since $y_{i} \in G\left(\mathbb{F}_{p}\right)$, we have $x_{i} \in \mathfrak{g}\left(\mathbb{F}_{p}\right)$. Set $\widehat{E}=\sigma\left(\mathbb{A}^{n}\right)$. 
Proposition 7. The map $\sigma$ is a homomorphism of algebraic groups with the following properties:

(a) $\sigma\left(\mathbb{A}^{n}\right)$ is an abelian algebraic subgroup of $G$;

(b) $\operatorname{Im}(\sigma) \subseteq \mathcal{U}^{\mathbb{F}_{p}}$ and $\operatorname{Im}(d \sigma) \subseteq \mathcal{N}^{\mathbb{F}}$;

(c) $\sigma: \mathbb{A}^{n} \rightarrow \widehat{E}$ is an isomorphism of algebraic varieties.

Proof. (a) It follows from Theorem 3(b) that $\left[x_{i}, x_{j}\right]=0$. So Proposition 6 shows that $\sigma$ is a homomorphism of algebraic groups and $\sigma\left(\mathbb{A}^{n}\right)$ is abelian.

(b) Since $\operatorname{Im}(\sigma)$ is an abelian subgroup of $G$ and $x_{1}, x_{2}, \ldots, x_{n} \in \mathfrak{g}\left(\mathbb{F}_{p}\right)$, it follows that $\operatorname{Im}(\sigma) \subseteq \mathcal{U}^{\mathbb{F}_{p}}$. Now consider the differential $d \sigma: \operatorname{Lie}\left(\mathbb{A}^{n}\right) \rightarrow \operatorname{Lie}(G)$ whose image is an abelian Lie subalgebra which is the $k$-linear span of the set $x_{1}, x_{2}, \ldots, x_{n}$. Therefore, $\operatorname{Im}(d \sigma) \subseteq \mathcal{N}^{\mathbb{F}_{p}}$.

(c) Suppose that $\operatorname{ker}(\sigma)$ has positive dimension. Then $x_{1}, \ldots, x_{n}$ would be linearly dependent over $k$. Since $x_{i} \in \mathfrak{g}\left(\mathbb{F}_{p}\right)$ for $1 \leq i \leq n$, the elements $x_{1}, \ldots, x_{n}$ would have to be linearly dependent over $\mathbb{F}_{p}$. Without loss of generality we may assume that $x_{1}$ is an $\mathbb{F}_{p}$-linear combination of $x_{2}, \ldots, x_{n}$. This shows that $y_{1}=$ $y_{2}^{a_{2}} \cdots y_{n}^{a_{n}}$ for some integers $a_{2}, \ldots, a_{n}$. This contradicts the fact that $y_{1}, \ldots, y_{n}$ are independent generators of $E$. Hence, $\mathbb{A}^{n} \rightarrow \sum_{i=1}^{n} k x_{i}$ is an isomorphism. Now the fact that $\sigma$ is an isomorphism of varieties follows from the isomorphism $\exp : \sum_{i=1}^{n} k x_{i} \rightarrow \hat{E}$.

4.2. For a given elementary abelian $p$-group $E$, we set $J=\operatorname{Rad}(k E)$, which is the ideal generated by $y-1$ for all $y \in E$. Note that $k E$ is the restricted enveloping algebra of the trivial restricted (abelian) Lie algebra $\left(J / J^{2}\right)$. The cohomological variety of $k E$ can be identified with $|E|_{k}=J / J^{2}$ [FP2], which is a $k$-vector space.

Let $E=\left\langle y_{1}\right\rangle \times\left\langle y_{2}\right\rangle \times \cdots \times\left\langle y_{n}\right\rangle$ for an independent generating set $\left\{y_{1}, \ldots, y_{n}\right\}$. Then $|E|_{k}$ can be identified with $k^{n}$ by $\left(t_{1}, t_{2}, \ldots, t_{n}\right) \mapsto \sum_{i=1}^{n} t_{i}\left(y_{i}-1\right)+J^{2}$. Under the above identification, for $M \in \bmod (E)$, the support variety of $M$ can be identified with [Ben1, 5.8.2, 5.8.3]

$$
|E|_{M}=\left\{\left(t_{1}, t_{2}, \ldots, t_{n}\right): M \text { is not free over }\left\langle 1+\sum_{i=1}^{n} t_{i}\left(y_{i}-1\right)\right\rangle\right\} \cup\{0\} .
$$

We say that $M$ is projective over $\langle x\rangle$ or $\left.M\right|_{\langle x\rangle}$ is projective if $M$ is projective over the group algebra $k\langle x\rangle$ when $x$ is an invertible element of order $p$ in a group ring or over the restricted enveloping algebra of the 1-dimensional Lie algebra $k x$ when $x$ is an element of the restricted Lie algebra with $x^{[p]}=0$.

For each $g \in G\left(\mathbb{F}_{p}\right), g E g^{-1}=\left\langle g y_{1} g^{-1}\right\rangle \times\left\langle g y_{2} g^{-1}\right\rangle \times \cdots \times\left\langle g y_{n} g^{-1}\right\rangle$ is an elementary abelian $p$-subgroup of rank $n$. Let $E^{\prime}=\left\langle u_{1}\right\rangle \times\left\langle u_{2}\right\rangle \times \cdots \times\left\langle u_{m}\right\rangle$ be another elementary abelian $p$-subgroup of $G\left(\mathbb{F}_{p}\right)$ such that $g E^{\prime} g^{-1} \subseteq E$ for some $g \in G\left(\mathbb{F}_{p}\right)$. We write $g u_{i} g^{-1}=\prod_{j=1}^{n} y_{j}^{r_{i j}}$. Then the matrix $\left(r_{i j}\right)$ has full rank over the field $\mathbb{F}_{p}$. For each $\left(t_{1}, \ldots, t_{n}\right) \in|E|_{k}$ and $u=1+\sum_{i=1}^{m} t_{i}\left(u_{i}-1\right) \in k E^{\prime}$ we have that

$$
g u g^{-1}=1+\sum_{i=1}^{m} t_{i}\left(g u_{i} g^{-1}-1\right)=1+\sum_{j=1}^{n}\left(\sum_{i=1}^{m} t_{i} r_{i j}\right)\left(y_{j}-1\right)+r_{2}
$$

where $r_{2}$ is an element in $J^{2}$. To prove the relation above we use the identity $(y z-1)=(y-1)+(z-1)+(y-1)(z-1)$. The conjugation map by $g: E^{\prime} \rightarrow E$ induces a map $\mathcal{S}(g):\left|E^{\prime}\right|_{k} \rightarrow|E|_{k}$. Under the given basis and the above identifications, the map $\mathcal{S}(g)$ is given by the matrix $\left(r_{i j}\right)$. Although the matrix description of the 
map depends on the choice of bases for $E^{\prime}$ and $E$, the map $\mathcal{S}(g)$ is independent of the bases.

Let $\mathcal{E}$ be the category whose objects are elementary abelian $p$-subgroups $E$ of the finite group $G\left(\mathbb{F}_{p}\right)$ and the morphism sets are $\left(E, E^{\prime}\right)=\left\{g \in G\left(\mathbb{F}_{p}\right): g E g^{-1} \subseteq\right.$ $\left.E^{\prime}\right\}$. Let $\mathcal{S}: \mathcal{E} \rightarrow \mathcal{V}$ be the functor that assigns to each $E$ the cohomological variety of $E$ where $\mathcal{V}$ is the category of all algebraic $k$-varieties. The morphism $g: E \rightarrow E^{\prime}$ induces a map $\mathcal{S}(g):|E|_{k} \rightarrow\left|E^{\prime}\right|_{k}$. Since the category $\mathcal{E}$ has only finitely many objects, the direct limit of the functor $\mathcal{S}$ exists in $\mathcal{V}$.

Let $\left|\widehat{G\left(\mathbb{F}_{p}\right)}\right|_{k}=\lim _{\mathcal{E}}|E|_{k}$ be the direct limit with the maps $\rho_{E}:|E|_{k} \rightarrow \lim _{\mathcal{E}}|E|_{k}$. This direct limit has the following universal property: if we are given an algebraic variety $V$ and any collection of morphisms $\phi_{E}:|E|_{k} \rightarrow V$ of algebraic varieties such that for every morphism $g: E \rightarrow E^{\prime}$ in $\mathcal{E}$ we have the following commutative diagram,

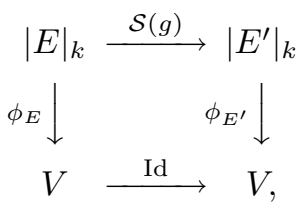

then there is a unique morphism of algebraic varieties $\psi: \underset{\lim _{\mathcal{E}}}{ }|E|_{k} \rightarrow V$ that makes the following diagram commute:

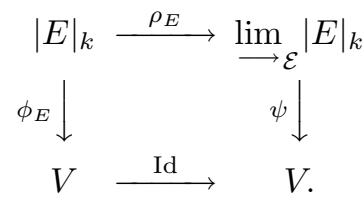

Given $E=\left\langle y_{1}\right\rangle \times\left\langle y_{2}\right\rangle \times \cdots \times\left\langle y_{n}\right\rangle$ and $x_{1}, x_{2}, \ldots, x_{n} \in \mathcal{N}^{\mathbb{F}_{p}}$ such that $\exp \left(x_{i}\right)=y_{i}$ for $i=1,2, \ldots, n$, define $\hat{\zeta}_{E}:|E|_{k} \rightarrow G$ by

$$
\hat{\zeta}_{E}\left(t_{1}, t_{2}, \ldots, t_{n}\right)=\prod_{i=1}^{n} \exp \left(t_{i} x_{i}\right) .
$$

Now let $\zeta_{E}:|E|_{k} \rightarrow G / G\left(\mathbb{F}_{p}\right)$ be the map defined as $\hat{\zeta}_{E}$ composed with the quotient map $\pi: G \rightarrow G / G\left(\mathbb{F}_{p}\right)$. The above discussion together with Theorem 3 shows that the map $\zeta_{E}$ is independent of the choice of basis for $E$. It follows from Proposition 7 that $\zeta_{E}$ is a finite map onto its image.

Let $\log : \mathcal{U}_{1} \rightarrow \mathcal{N}_{1}$ be the inverse morphism of exp and $\overline{\log }: \mathcal{U}_{1} / G\left(\mathbb{F}_{p}\right) \rightarrow$ $\mathcal{N}_{1} / G\left(\mathbb{F}_{p}\right)$ be the induced morphism (see Theorem 3 ).

Theorem 5. Let $G$ be a simple algebraic group and assume that $p$ is good, $\mathcal{N}_{1}$ is normal, and the Assumption 1 is satisfied. There are finite surjective maps of varieties $\phi:\left|\widehat{G\left(\mathbb{F}_{p}\right)}\right|_{k} \rightarrow \mathcal{U}^{\mathbb{F}_{p}} / G\left(\mathbb{F}_{p}\right)$ and $\psi:\left|\widehat{G\left(\mathbb{F}_{p}\right)}\right|_{k} \rightarrow \mathcal{N}^{\mathbb{F}_{p}} / G\left(\mathbb{F}_{p}\right)$ such that $\psi=\overline{\log } \circ \phi$ and $\phi \circ \rho_{E}=\zeta_{E}$ for each elementary abelian subgroup $E$ of $G\left(\mathbb{F}_{p}\right)$.

Proof. The finite group $G\left(\mathbb{F}_{p}\right)$ acts on the algebraic group $G$ by conjugation. Let $E=\left\langle y_{1}\right\rangle \times\left\langle y_{2}\right\rangle \times \cdots \times\left\langle y_{n}\right\rangle$ and $E^{\prime}=\left\langle y_{1}^{\prime}\right\rangle \times\left\langle y_{2}^{\prime}\right\rangle \times \cdots \times\left\langle y_{m}^{\prime}\right\rangle$ be elementary abelian $p$-subgroups of $G\left(\mathbb{F}_{p}\right)$ with $g E^{\prime} g^{-1} \subseteq E$ for some $g \in G\left(\mathbb{F}_{p}\right)$. Let $y_{i}=\exp \left(x_{i}\right)$ for $i=1,2, \ldots, n$ and $y_{j}^{\prime}=\exp \left(x_{j}^{\prime}\right)$ for $j=1,2, \ldots, m$ where $x_{i}, x_{j}^{\prime} \in \mathfrak{g}_{\mathbb{F}_{p}}$ for all $i, j$. Under the adjoint action of $G$ on $\mathfrak{g}, \operatorname{Ad}(g)\left(x_{i}^{\prime}\right)=\sum_{j=1}^{n} r_{i j} x_{j}$. Then for each 
$\left(t_{1}, t_{2}, \ldots, t_{m}\right) \in\left|E^{\prime}\right|_{k}$,

$$
\begin{array}{r}
\pi\left(\hat{\zeta}_{E^{\prime}}\left(t_{1}, \ldots, t_{m}\right)\right)=\pi\left(g \cdot \hat{\zeta}_{E^{\prime}}\left(t_{1}, \ldots, t_{m}\right)\right)=\pi\left(g\left(\prod_{i=1}^{m} \exp \left(t_{i} x_{i}^{\prime}\right)\right) g^{-1}\right) \\
=\pi\left(\prod_{i=1}^{n} \exp \left(\sum_{i=1}^{n} t_{i} r_{i j} x_{j}\right)\right)=\zeta_{E}\left(\mathcal{S}(g)\left(t_{1}, t_{2}, \ldots, t_{m}\right)\right) .
\end{array}
$$

The above identity proves that the diagram

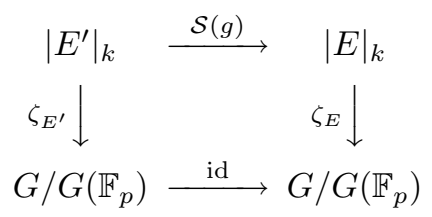

commutes for all $g \in G\left(\mathbb{F}_{p}\right)$. The horizontal map on the bottom of the diagram is induced by the conjugation of $g$ and thus is the identity map on $G / G\left(\mathbb{F}_{p}\right)$.

Hence, by the universal property of the direct limit, there exists a map $\phi$ : $\lim _{E}|E|_{k} \rightarrow G / G\left(\mathbb{F}_{p}\right)$. The image of each $\zeta_{E}$ is in $\mathcal{U}^{\mathbb{F}_{p}} ;$ thus $\phi: \lim _{E}|E|_{k} \rightarrow$ $\mathcal{U}^{\mathbb{F}_{p}} / G\left(\mathbb{F}_{p}\right)$ is defined. The map $\phi$ is onto since, for each $u \in \mathcal{U}^{\mathbb{F}_{p}}$, there exists an elementary abelian $p$-subgroup $E$ such that $\hat{\zeta}_{E}\left(|E|_{k}\right)$ contains $u$. The map $\phi$ is finite because there are only finitely many elementary abelian $p$-subgroups and each $\zeta_{E}$ is finite. Since $\log$ is a $G\left(\mathbb{F}_{p}\right)$-isomorphism of $\mathcal{U}^{\mathbb{F}_{p}}$ with $\mathcal{N}^{\mathbb{F}_{p}}$, the map log induces the isomorphism $\overline{\log }$ between the quotient spaces $\mathcal{U}^{\mathbb{F}_{p}} / G\left(\mathbb{F}_{p}\right)$ and $\mathcal{N}^{\mathbb{F}_{p}} / G\left(\mathbb{F}_{p}\right)$. We define $\psi=\overline{\log } \circ \phi$ with $\psi:\left|\widehat{G\left(\mathbb{F}_{p}\right)}\right|_{k} \rightarrow \mathcal{N}^{\mathbb{F}_{p}} / G\left(\mathbb{F}_{p}\right)$. The finiteness of $\psi$ follows from the finiteness of $\phi$.

We remark that, by Quillen's Stratification Theorem [Ben1, Cor 5.6.4], $\left|G\left(\mathbb{F}_{p}\right)\right|_{k}$ is homeomorphic to $\left|\widehat{G\left(\mathbb{F}_{p}\right)}\right|_{k}=\lim _{\longrightarrow E}|E|_{k}$. For the sake of convenience, we define our maps $\phi$ and $\psi$ explicitly on $\left|\widehat{G\left(\mathbb{F}_{p}\right)}\right|_{k}$ rather than on $\left|G\left(\mathbb{F}_{p}\right)\right|_{k}$.

4.3. For $M \in \bmod \left(G\left(\mathbb{F}_{p}\right)\right)$, let $\left|\widehat{G\left(\mathbb{F}_{p}\right)}\right|_{M}=\lim _{E}|E|_{M}$. Observe that $\left|\widehat{G\left(\mathbb{F}_{p}\right)}\right|_{M}$ is a closed subvariety of $\left|\widehat{G\left(\mathbb{F}_{p}\right)}\right|_{k}$. By a well-known result of Avrunin and Scott [AS], the restriction maps induce an inseparable isogeny $\delta:\left|\widehat{G\left(\mathbb{F}_{p}\right)}\right|_{M} \rightarrow\left|G\left(\mathbb{F}_{p}\right)\right|_{M}$. A fundamental question is to describe the image of $\left|\widehat{G\left(\mathbb{F}_{p}\right)}\right|_{M}$ under the maps $\phi$ and $\psi$. We begin this investigation by proving a result which compares restrictions of nilpotent (and unipotent) elements on a rational $G$-module $M$.

Proposition 8. Let $M$ be a finite dimensional $k$-vector space and $x, y \in \operatorname{End}_{k}(M)$ be two commuting elements such that $x \neq 0$ and $x^{p}=y^{p-1}=0$. Then

(a) $1+x$ and $1+x+y$ are invertible, and

(b) if $M$ is a free module over the group algebra $k\langle 1+x\rangle$, then $M$ is free over the group algebra $k\langle 1+x+y\rangle$.

Proof. Since $x^{p}=0$ and $x \neq 0$, the element $1+x$ is invertible of order $p$ in $\operatorname{End}_{k}(M)$. It is clear that $1+x+y$ is invertible. There exists an isomorphism of algebras $k\langle 1+x\rangle \cong k[T] /\left\langle T^{p}\right\rangle$ where $1+x \mapsto 1+T$. Since $M$ is free as a module over $k\langle 1+x\rangle, M$ is free over $k[T] /\left\langle T^{p}\right\rangle$. Let $\left\{v_{1}, \ldots, v_{r}\right\}$ be a free basis. Then $\left\{v_{i}, T v_{i}, \ldots, T^{p-1} v_{i} \mid i=1, \ldots, r\right\}$ is a $k$-basis of $M$. In particular $x^{p-1} \neq 0$. 
Let $\mathfrak{l}$ be a two dimensional trivial restricted Lie algebra with basis $\left\{z_{1}, z_{2}\right\}$. Define a restricted l-module structure on $M$ by $z_{1} \mapsto x$ and $z_{2} \mapsto y$. Note that the support variety of $M$ as an l-module [FP2] is

$$
|M|_{\mathfrak{r}}=\left\{z \in \mathfrak{l}|M|_{\langle z\rangle} \text { is not projective }\right\} \cup\{0\} .
$$

Let $M=M_{1} \oplus M_{2} \oplus \cdots \oplus M_{s}$ be a decomposition of $M$ into indecomposable $u(\mathfrak{l})$ modules. Note that $M$ is free over $\langle z\rangle$ if and only if $M_{j}$ is free over $\langle z\rangle$ for all $j$. So without a loss of generality we may assume that $M$ is indecomposable over $u(\mathfrak{l})$.

By our assumption $y^{p-1}=0$, so the above characterization of the support variety of $M$ shows that $z_{2} \in|M|_{\mathfrak{l}}$ while $z_{1} \notin|M|_{\mathfrak{l}}$. Consequently, $|M|_{\mathfrak{l}}$ is a proper closed subvariety of $\mathfrak{l}$. The projective variety $\operatorname{Proj}\left(|M|_{\mathfrak{l}}\right)$ is a proper closed connected subvariety of $\mathbb{P}^{1}[\mathrm{FP} 2]$ and is either empty or a single point. Hence, $|M|_{\mathfrak{r}}$ is at most a 1 -dimensional vector subspace of $\mathfrak{l}$ spanned by the line $k z_{1}$. It follows that $z_{1}+z_{2} \notin|M|_{\mathfrak{r}}$ since $z_{2} \in|M|_{\mathfrak{r}}$ and $z_{1} \notin|M|_{\mathfrak{l}}$. Therefore, $M$ is a projective module for the algebra $u\left(\left\langle z_{1}+z_{2}\right\rangle\right)$ and the proposition follows. ${ }^{1}$

4.4. Let $\sigma: \mathrm{SL}_{2} \rightarrow G$ be a homomorphism of algebraic groups defined over the localization $\mathbb{Z}_{(p)}$ of $\mathbb{Z}$ at $p$. The differential $d \sigma: \operatorname{Dist}\left(\mathrm{SL}_{2}\right) \rightarrow \operatorname{Dist}(G)$ is a homomorphism of associative algebras. Note that the distribution algebra of the unipotent subgroup $1+k E_{21}$ of $\mathrm{SL}_{2}$ has a basis $E_{21}^{(n)}$ satisfying $E_{21}^{(n)} E_{21}^{(m)}=\left(\begin{array}{c}n+m \\ n\end{array}\right) E_{21}^{(n+m)}$. Let $x \in \mathfrak{g}$ such that $x=d \sigma\left(E_{12}\right)$. We set $x^{(n)}=d \sigma\left(E_{12}^{(n)}\right) \in \operatorname{Dist}(G)$.

For any rational $G$-module $M$, the action of $\exp (t x)$ is the same as $\sum_{n \geq 0} t^{n} x^{(n)}$ by Proposition 2. Note that $x^{(n)} x^{(m)}=\left(\begin{array}{c}n+m \\ n\end{array}\right) x^{(n+m)}$ (see [St]). For $n=\sum_{i} r_{i} p^{i}$ with $0 \leq r_{i}<p$, we have that $x^{(n)}=\prod_{i}\left(x^{\left(p^{i}\right)}\right)^{r_{i}} / r_{i}$ !. Let $b_{s}=\sum_{i=0}^{s} r_{i} p^{i}$. By considering the images in $\operatorname{End}_{k}(M)$, we have

$$
\exp (t x)=\sum_{s \geq 0} \sum_{0 \leq r_{0}, \ldots, r_{s}<p} \frac{x^{r_{0}}\left(x^{(p)}\right)^{r_{1}} \cdots\left(x^{\left(p^{s}\right)}\right)^{r_{s}}}{r_{0} ! r_{1} ! \cdots r_{s} !} t^{b_{s}} .
$$

4.5. Let $x \in \mathcal{N}_{1}$. There is a homomorphism of $\sigma: \mathrm{SL}_{2} \rightarrow G$ such that $x=d \sigma\left(E_{21}\right)$. As in Section 4.4, $x^{(n)} \in \operatorname{Dist}(G)$ is defined with fixed $\sigma$. The following proposition will provide some motivation for the results in the following two subsections.

Proposition 9. Let $M \in \bmod (G)$. Suppose $x$ satisfies $\left(x^{\left(p^{i}\right)}\right)^{p-1}=0$ in $\operatorname{End}_{k}(M)$ for all $i \geq 1$. Then for each $t \in k^{*},\left.M\right|_{\langle\exp (t x)\rangle}$ is free if and only if $\left.M\right|_{\langle x\rangle}$ is free.

Proof. Note that in $\operatorname{End}_{k}(M)$ we can write $\exp (t x)=1+t x+y$ such that $t x$ and $y$ satisfy the condition of Proposition 8. Since $t \in k^{*},\left.M\right|_{\langle x\rangle}$ is free implies that $\left.M\right|_{\langle 1+t x\rangle}$ is free. Therefore, by Proposition $8,\left.M\right|_{\langle\exp (t x)\rangle}$ is free. Conversely, we can substitute $t x+y$ for $x$ and $-y$ for $y$ in Proposition 8. Then the same argument shows that if $\left.M\right|_{\langle\exp (t x)\rangle}$ is free, then $\left.M\right|_{\langle x\rangle}$ is free.

When $G=\mathrm{SL}_{2}$ and $x$ is a positive root vector, the condition of the above proposition is satisfied for any $G$-module $M$ all of whose weights are strictly less than $p(p-1)$. Therefore, under these conditions, $M$ is projective over the Sylow $p$-subgroup of $\mathrm{SL}_{2}\left(\mathbb{F}_{p}\right)$ if and only if it is projective over the nilpotent radical of the Borel subalgebra. Consequently, $M$ is projective as an $\mathrm{SL}_{2}\left(\mathbb{F}_{p}\right)$-module if and only if

\footnotetext{
${ }^{1}$ We thank Julia Pevtsova for pointing out a gap in the earlier proof of (b). The earlier version relied on the proof of [Ben2, p. 129, Lemma 2.25.5], which is not correct because the subgroup of upper triangular matrices in the centralizer contains a Sylow $p$-subgroup if and only if there is only one $p \times p$ Jordan block.
} 
$M$ is projective for the Lie algebra $\mathfrak{s l}_{2}$. This result was proved in [LN, Thm. 4.3] for $\mathrm{SL}_{2}$ by classifying all indecomposable $\mathrm{SL}_{2}$-modules with weights less than $p(p-1)$ and computing their dimensions. It turns out that the indecomposable $\mathrm{SL}_{2}$-modules with weights less than $p(p-1)$ and dimension divisible by $p$ are projective over $\mathfrak{s l}_{2}$. The aforementioned proposition provides a much more natural (and enlightening) proof of this result.

4.6. The category $\mathcal{C}_{p}$. Let $G$ be a simple algebraic group of Lie rank $l$, and let $\mathcal{C}_{p}$ be the full subcategory of $G$-modules for which all dominant weights $\lambda$ satisfy the condition

$$
\sum_{i=1}^{l} \sum_{j=1}^{l}\left\langle\lambda, \alpha_{i}^{\vee}\right\rangle b_{i j}<p(p-1) / 2 .
$$

Here the matrix $\left(b_{i j}\right)$ is the inverse matrix of the Cartan matrix $\left(\left\langle\alpha_{i}, \alpha_{j}^{\vee}\right\rangle\right)$. Let $\omega_{1}, \omega_{2}, \ldots, \omega_{l}$ be the fundamental weights. Note that $\omega_{i}=\sum_{j=1}^{l} b_{i j} \alpha_{j}$, and

$$
\omega_{i}^{\vee}=2 \omega_{i} /\left\langle\alpha_{i}, \alpha_{i}\right\rangle=\sum_{j=1}^{l} b_{j i} \alpha_{j}^{\vee}
$$

satisfies the condition that $\lambda=\sum_{i=1}^{l}\left\langle\lambda, \omega_{i}^{\vee}\right\rangle \alpha_{i}$ for any $\lambda \in X(T)$. Hence the left hand side of (4.6.1) equals $\sum_{j=1}^{l}\left\langle\lambda, \omega_{j}^{\vee}\right\rangle$. For a simple algebraic group $G$ let $c$ be the value given in the table below.

\begin{tabular}{|c|c|c|c|c|c|c|c|c|c|}
\hline type & $A_{l}$ & $B_{l}$ & $C_{l}$ & $D_{l}$ & $E_{6}$ & $E_{7}$ & $E_{8}$ & $F_{4}$ & $G_{2}$ \\
\hline$c$ & $((l+1) / 2)^{2}$ & $l(l+1) / 2$ & $l^{2} / 2$ & $l(l-1) / 2$ & 16 & 27 & 46 & 11 & 4 \\
\hline
\end{tabular}

The condition $\left\langle\lambda, \alpha_{0}^{\vee}\right\rangle\left\langle p(p-1) / c\right.$, where $\alpha_{0}$ is the highest short root of the indecomposable root system, will insure that $\lambda$ satisfies the condition (4.6.1). The computation of $c$ is provided in the Appendix in $\S 6$.

Let $x \in \mathcal{N}^{\mathbb{F}_{p}}$. We have $x=t_{1} x_{1}+t_{2} x_{2}+\cdots+t_{n} x_{n}$ where $x_{i} \in \mathfrak{g}\left(\mathbb{F}_{p}\right)$, and $\left[x_{i}, x_{j}\right]=$ 0 . Let $1 \leq i \leq n$ be fixed. According to [Sei], there exists a homomorphism $\sigma: \mathrm{SL}_{2} \rightarrow G$ of algebraic groups such that $d \sigma\left(E_{12}\right)=x_{i}$ (cf. $\left.\S 2.6\right)$. Let $T^{\prime}$ be the maximal torus of $\mathrm{SL}_{2}$ and $T$ be the maximal torus in $G$. Since $M$ is a $G$-module, we can conjugate $x_{i}$ and assume that $x_{i} \in \mathfrak{n}_{J}$ and the image of $T^{\prime}$ is contained in $T$. Since $\operatorname{hom}\left(T^{\prime}, T\right)=\operatorname{hom}\left(G_{m}, T\right)=\operatorname{hom}_{\mathbb{Z}}(X(T), \mathbb{Z})$, the homomorphism $\sigma: T^{\prime} \rightarrow T$ determines an element $t^{\vee} \in \operatorname{hom}_{\mathbb{Z}}(X(T), \mathbb{Z})$. On any $G$-module, $T^{\prime}$ acts on the weight space that has $T$-weight $\lambda$ by the $T^{\prime}$-weight $\left\langle\lambda, t^{\vee}\right\rangle=t^{\vee}(\lambda)$. By [C, Lemma 5.6.5], we can choose a Weyl group element conjugation to assume that $t^{\vee}(\alpha) \geq 0$ for all $\alpha \in \Phi^{+}$.

By Dynkin's theorem [C, Prop 5.6.6], the possible values for $a_{j}=t^{\vee}\left(\alpha_{j}\right)$ are $0,1,2$. For any weight $\lambda \in X(T)$, write $\lambda=\sum_{j=1}^{l}\left\langle\lambda, \omega_{j}^{\vee}\right\rangle \alpha_{j}$. Consequently, for any dominant weight $\lambda$ of the $G$-module $M$ in $\mathcal{C}_{p}$, we have that

$$
t^{\vee}(\lambda)=\sum_{j=1}^{l}\left\langle\lambda, \omega_{j}^{\vee}\right\rangle t^{\vee}\left(\alpha_{j}\right) \leq \sum_{j=1}^{l} 2\left\langle\lambda, \omega_{j}^{\vee}\right\rangle<p(p-1) .
$$

Note that the above condition is equivalent to the statement that $t^{\vee}\left(w_{0}(\lambda)\right)>$ $-p(p-1)$ where $w_{0}$ is the longest element of the Weyl group. Thus for any $M \in \mathcal{C}_{p}$ and any weight $\mu$ of $M$, there is a dominant weight $\lambda$ of $M$ in $W \mu$ such that $w_{0}(\lambda) \leq \mu \leq \lambda$ in the usual partial order. Since $t^{\vee}(\nu) \geq 0$ for all $\nu \in \mathbb{N} \Phi^{+}$, we have 
$p(p-1)>t^{\vee}(\mu)>-p(p-1)$. Note that each $x^{\left(p^{s}\right)}$ has $T^{\prime}$-weight $2 p^{s}$. If $v \in M$ has $T$-weight $\mu$, then $\left(x^{\left(p^{s}\right)}\right)^{p-1} v \neq 0$ would have $T^{\prime}$-weight $2(p-1) p^{s}+t^{\vee}(\mu) \geq p(p-1)$, assuming that $s>0$. Thus, $\left(x^{\left(p^{s}\right)}\right)^{p-1} v=0$, and $\left(x^{\left(p^{s}\right)}\right)^{p-1} M=0$.

4.7. Let us fix some notation for the rest of this section. Let $x=t_{1} x_{1}+t_{2} x_{2}+\cdots+$ $t_{n} x_{n} \in \mathcal{N}^{\mathbb{F}_{p}}$ where $x_{1}, \ldots, x_{n} \in \mathfrak{g}\left(\mathbb{F}_{p}\right)$ and $t_{1}, \ldots, t_{n} \in k$. Assume that $\left[x_{i}, x_{j}\right]=0$ and $x_{i}^{[p]}=0$. Set $u=\prod_{i=1}^{n} \exp \left(t_{i} x_{i}\right) \in \mathcal{U}^{\mathbb{F}_{p}}$ and $y=1+\sum_{i=1}^{n} t_{i}\left(y_{i}-1\right) \in$ $k G\left(\mathbb{F}_{p}\right)$ where $y_{i}=\exp \left(x_{i}\right)$ for $i=1,2, \ldots, n$. Recall that under these conditions: $\phi\left(t_{1}, t_{2}, \ldots, t_{n}\right)=u$ and $\psi\left(t_{1}, t_{2}, \ldots, t_{n}\right)=x$.

Theorem 6. Assume that $G$ satisfies the conditions at the beginning of Section 4. Let $M \in \bmod (G)$ such that $\left(x_{i}^{\left(p^{s}\right)}\right)^{p-1}=0$ in $\operatorname{End}_{k} M$ for all $s \geq 1$ (i.e. if $M$ is in $\left.\mathcal{C}_{p}\right)$. Then the following three conditions are equivalent.

(a) $\left.M\right|_{\langle x\rangle}$ is not free.

(b) $\left.M\right|_{\langle u\rangle}$ is not free.

(c) $\left.M\right|_{\langle y\rangle}$ is not free.

Proof. $(a) \Leftrightarrow(c)$. First observe that as operators in $\operatorname{End}_{k}(M)$,

$$
y-1=\sum_{i=1}^{n} t_{i} x_{i}+\sum_{i=1}^{n} \sum_{j \geq 2} t_{i} x_{i}^{(j)}=x+\sum_{i=1}^{n} \sum_{j \geq 2} t_{i} x_{i}^{(j)} .
$$

Since $x_{i}^{(j)}$ are all commuting elements in $\operatorname{End}_{k}(M)$, the assumption shows that $\left(x_{i}^{(j)}\right)^{p-1}=0$ for all $j \geq 2$. Applying Proposition 8 repeatedly by adding the terms $t_{i} x_{i}^{(j)}$, we see that $\left.M\right|_{\langle y\rangle}$ is free whenever $\left.M\right|_{\langle x\rangle}$ is free. Conversely, assume that $\left.M\right|_{\langle y\rangle}$ is free. Note that $(y-1)^{p}=0$ in $\operatorname{End}_{k}(M)$. By adding $-t_{i} x_{i}^{(j)}$ for $j \geq 2$ we obtain that $\left.M\right|_{\langle x\rangle}$ is free, from Proposition 8 .

$(a) \Leftrightarrow(b)$. An expansion of $u$ as an operator in $\operatorname{End}_{k}(M)$ has the form

$$
u=1+x+\sum_{j_{1}, j_{2}, \ldots, j_{n}} \prod_{i=1}^{n} t_{i}^{j_{i}} x_{i}^{\left(j_{i}\right)} .
$$

The summation is over all $\left(j_{1}, j_{2}, \ldots, j_{n}\right) \in \mathbb{N}^{n}$ such that $\sum_{i=1}^{n} j_{i} \geq 2$. Since all factors in (4.6.2) commute in $\operatorname{End}_{k}(M)$, we have

$$
\left(\prod_{i=1}^{n} t_{i}^{j_{i}} x_{i}^{\left(j_{i}\right)}\right)^{p-1}=0
$$

by the assumption. Then the same argument as above shows that $\left.M\right|_{\langle x\rangle}$ is free if and only if $\left.M\right|_{\langle u\rangle}$ is free.

4.8. We now apply Proposition 8 to describe the image of $\left|\widehat{G\left(\mathbb{F}_{p}\right)}\right|_{M}$ in $\mathcal{U}^{\mathbb{F}_{p}} / G\left(\mathbb{F}_{p}\right)$ $\left(\right.$ resp. $\left.\mathcal{N}^{\mathbb{F}_{p}} / G\left(\mathbb{F}_{p}\right)\right)$ under $\phi($ resp. $\psi)$.

Corollary 1. Let $G$ be a simple algebraic group such that $p$ is good, $\mathcal{N}_{1}$ is normal, and Assumption 1 holds and let $M \in \mathcal{C}_{p}$. Then

(a) $\left\{u \in \mathcal{U}^{\mathbb{F}_{p}}:\left.M\right|_{\langle u\rangle}\right.$ is not free $\}=\exp \left(\left\{x \in \mathcal{N}^{\mathbb{F}_{p}}:\left.M\right|_{\langle x\rangle}\right.\right.$ is not free $\left.\}\right)$.

(b) $\phi\left(\left|\widehat{G\left(\mathbb{F}_{p}\right)}\right|_{M}\right)=\left\{u \in \mathcal{U}^{\mathbb{F}_{p}}:\left.\quad M\right|_{\langle u\rangle}\right.$ is not free $\} / G\left(\mathbb{F}_{p}\right) \cup\{0\}$.

(c) $\psi\left(\left|\widehat{G\left(\mathbb{F}_{p}\right)}\right|_{M}\right)=\left\{x \in \mathcal{N}^{\mathbb{F}_{p}}: x^{[p]}=0,\left.\quad M\right|_{\langle x\rangle}\right.$ is not free $\} / G\left(\mathbb{F}_{p}\right) \cup\{0\}$. 
Proof. Part (a) follows directly from the equivalence of (a) and (b) in Theorem 6. Part (b) can be seen by using the equivalence of (b) and (c) of Theorem 6. Finally, part (c) holds because of (a) and (c) of Theorem 6 .

4.9. A precise relationship between $\left|G\left(\mathbb{F}_{p}\right)\right|_{M}$ and $\left|G_{1}\right|_{M}$ can now be provided when $M$ is a module in $\mathcal{C}_{p}$.

Corollary 2. Let $G$ be a simple algebraic group and assume that $p$ satisfies the assumptions at the beginning of Section 4. If $M$ is in $\mathcal{C}_{p}$, then $\psi\left(\left|\widehat{G\left(\mathbb{F}_{p}\right)}\right|_{M}\right)=$ $\left(\mathcal{N}_{1}^{\mathbb{F}_{p}} \cap|\mathfrak{g}|_{M}\right) / G\left(\mathbb{F}_{p}\right)$. Furthermore,

$$
\operatorname{dim}\left|G\left(\mathbb{F}_{p}\right)\right|_{M}=\operatorname{dim}\left(\mathcal{N}^{\mathbb{F}} \cap|\mathfrak{g}|_{M}\right) .
$$

Proof. The first statement follows from Corollary 1(c), and the equality of dimensions holds because $\psi$ is a finite map.

For $M$ in $\mathcal{C}_{p}$, the preceding result provides a complete answer to the question (1.1.2) raised by Parshall. This relationship will be used in Section 5 to compute the complexity of modules for the finite group $G\left(\mathbb{F}_{p}\right)$. We should also add that for $M \in \bmod (G),|\mathfrak{g}|_{M}$ is a closed $G$-invariant subvariety of $\mathcal{N}_{1}$ and thus the union of (finitely many) nilpotent orbits. An interesting and useful problem would be to determine the dimension of the intersection of a nilpotent orbit with $\mathcal{N}^{\mathbb{F}}$.

\section{Applications}

5.1. Alperin and Mason [AM2] proved that for a finite group $G\left(\mathbb{F}_{q}\right)$ with underlying root system of type $A, D$ or $E$, a simple module $S$ is isomorphic to the Steinberg module if and only if $S$ is projective upon restriction to a root subgroup $X_{\alpha}(t)$. Our first application extends this result to all root systems in the case when $q=p$ and $p \geq h$.

Theorem 7. Let $G$ be a simple algebraic group with $p \geq h$. Then a simple module $S$ for $G\left(\mathbb{F}_{p}\right)$ is the Steinberg module if and only if $\left.S\right|_{X_{\alpha}(t)}$ is projective for some long root $\alpha$.

Proof. If $S$ is the Steinberg module, then it is projective over $G\left(\mathbb{F}_{p}\right)$ and thus projective over all root subgroups.

On the other hand, suppose that $\left.S\right|_{X_{\alpha}(t)}$ is projective for some long root $\alpha$. Since all long roots are conjugate via the Weyl group action, we may assume that $\alpha=-\tilde{\alpha}$, where $\tilde{\alpha}$ is the highest root. Let $S=L(\lambda)$ where $\lambda \in X_{1}(T)$. Since $p \geq h$, for all weights $\mu$ of $L(\lambda)$ we have

$$
\left\langle\mu, \tilde{\alpha}^{\vee}\right\rangle \leq\left\langle\lambda, \alpha_{0}^{\vee}\right\rangle \leq\left\langle(p-1) \rho, \alpha_{0}^{\vee}\right\rangle<p(p-1),
$$

where $\alpha_{0}$ is the highest short root. Now one can use a weight argument to show that $\left(x_{\alpha}^{\left(p^{s}\right)}\right)^{p-1}$ acts as zero on $S$ for $s \geq 1$ (since $\mu=w_{0}\left(\lambda+(p-1) p^{s} \alpha\right.$ ) is not a weight of $L(\lambda))$. It follows by Proposition 4.5 that $S$ must be projective over $\left\langle x_{\alpha}\right\rangle$, and projective over $G_{1}$ by [FP2, (2.4)(b) Prop.]. This proves the conclusion because the only simple projective module over $G_{1}$ is the Steinberg module. 
5.2. Janiszczak and Jantzen $[\mathrm{JJ}]$ proved that there are no periodic nonprojective simple modules for a group $G\left(\mathbb{F}_{q}\right)$ when $\operatorname{rank}(G) \geq 2$. The following results demonstrate how the map $\psi$ and the geometry of nilpotent orbits can be used to extend this result in several different directions because all simple $G\left(\mathbb{F}_{p}\right)$-modules are in $\mathcal{C}_{p}$.

Proposition 10. Let $G=\mathrm{GL}_{n+1}(k)$ ( $\Phi$ of type $\left.A_{n}\right)$. Then $\operatorname{dim}\left(\overline{\mathcal{O}} \cap \mathcal{N}^{\mathbb{F}_{p}}\right) \geq n$ for any nonzero nilpotent orbit $\mathcal{O}$. Furthermore, if $G$ is simple of rank at least two and if $\mathcal{O}$ is the minimal nilpotent orbit, then $\operatorname{dim}\left(\overline{\mathcal{O}} \cap \mathcal{N}^{\mathbb{F}_{p}}\right) \geq 2$.

Proof. We first assume that $G$ is of type $A_{n}$. Then every nilpotent orbit is a Richardson orbit [Hum4, 5.5]. The minimal orbit corresponds to a maximal parabolic subalgebra which has a nilpotent radical, that is, an $n$-dimensional abelian subalgebra with a basis consisting of root vectors in $\mathfrak{g}\left(\mathbb{F}_{p}\right)$. This shows that $\operatorname{dim}\left(\overline{\mathcal{O}} \cap \mathcal{N}^{\mathbb{F}} p\right) \geq n$.

Note that for another simple algebraic group $G$ of any type other than $C_{n}(n \geq$ 2), $G$ contains a rank 2 subgroup $K$ defined over $\mathbb{F}_{p}$ with root system $A_{2}$ consisting of some long roots of $G$. The minimal orbit of $G$ is the orbit of a long root vector. Thus $\mathcal{O} \cap K$ contains the minimal nilpotent orbit of $K$. Note that $\operatorname{Lie}\left(K_{\mathbb{F}_{p}}\right) \subseteq \mathfrak{g}_{\mathbb{F}_{p}}$. Thus the lemma follows from the result for type $A_{2}$. For type $C_{n}$, it suffices to consider the case when $n=2$ and then apply the same reasoning as above. In this case $C_{2}=B_{2}$. Let $\alpha, \beta$ be the simple roots with $\beta$ a long root. A quick computation shows that $\exp \left(x_{\alpha}\right)\left(x_{\beta}\right)=x_{\beta}+x_{\beta+\alpha}+x_{\beta+2 \alpha} \in \mathcal{O}$. Now the actions of the maximal tori of the two type $A_{1}$ subgroups corresponding to the long roots $\beta$ and $\beta+2 \alpha$ yield that $t^{2} x_{\beta}+t s x_{\beta+\alpha}+s^{2} x_{\beta+2 \alpha} \in \mathcal{O}$ for all $t, s \in k^{*}$. Note that the set of all these elements is in $\mathcal{N}^{\mathbb{F}}$ and is a two dimensional closed subvariety in $k x_{\beta}+k x_{\beta+\alpha}+k x_{\beta+2 \alpha} \subseteq \mathcal{N}^{\mathbb{F}_{p}}$. This proves the result for groups of type $C_{n}$.

Corollary 3. Let $G$ be a simple algebraic group and let $M \in \mathcal{C}_{p}$ be a $G$-module which is not projective as a $G\left(\mathbb{F}_{p}\right)$-module.

(a) If $M$ is indecomposable in $\bmod \left(G\left(\mathbb{F}_{p}\right)\right)$ and $\operatorname{rank}(G) \geq 2$, then $\operatorname{dim}\left|G\left(\mathbb{F}_{p}\right)\right|_{M}$ $\geq 2$.

(b) If $G$ has an underlying root system of type $A_{n}$, then $\operatorname{dim}\left|G\left(\mathbb{F}_{p}\right)\right|_{M} \geq n$.

Proof. For (a), suppose that $M$ is a nonprojective indecomposable module over $G\left(\mathbb{F}_{p}\right)$. Then $M$ is nonprojective over $\mathfrak{g}$ by [LN, Cor. 3.5]. Therefore, $G \cdot x_{\beta} \subseteq|\mathfrak{g}|_{M}$ for $\beta$ the highest long root vector. Now the result follows from Proposition 10 and Corollary 2. Part (b) is a direct consequence of Proposition 10.

It is interesting to note that for simple algebraic groups with root systems other than of type $A_{n}$, the minimal orbit is rigid [CM, Lem. 7.1.5], and thus cannot be Richardson.

5.3. Rank 2 groups. For $\lambda \in X(T)$, let $\Phi_{\lambda}=\left\{\alpha \in \Phi^{+}:\left\langle\lambda+\rho, \alpha^{\vee}\right\rangle \in p \mathbb{Z}\right\}$. When $p$ is good, there exists a subset $J$ of simple roots and $w \in W$ such that $w\left(\Phi_{\lambda}\right)=\Phi_{J}$. Let $\mathfrak{u}_{J}$ be the nilpotent radical of the parabolic algebra determined by $J$. In [NPV, (6.6.1) Cor.] the support varieties of simple modules for $\mathfrak{g}$ were calculated for rank 2 groups. The next result shows how to use this information to calculate the dimensions of the support varieties for simple modules over $G\left(\mathbb{F}_{p}\right)$ when $G$ has rank two. 
Theorem 8. Let $G$ be a simple algebraic group of rank 2 with underlying root system $A_{2}$ (resp. $B_{2}$ or $G_{2}$ ) with $p>3$ (resp. $p>7$, or $p>19$ ) and let $\lambda \in X_{1}(T)$.

(a) If $\lambda=(p-1) \rho$, then $\operatorname{dim}\left|G\left(\mathbb{F}_{p}\right)\right|_{L(\lambda)}=0$.

(b) If $\lambda \neq(p-1) \rho$, then $\operatorname{dim}\left|G\left(\mathbb{F}_{p}\right)\right|_{L(\lambda)}= \begin{cases}2 & \text { for } A_{2}, \\ 3 & \text { for } B_{2} \text { and } G_{2} \text {. }\end{cases}$

Proof. (a) Observe that $\lambda=(p-1) \rho$ if and only if $\Phi_{\lambda}=\Phi$. Furthermore, $L((p-1) \rho)$ is the Steinberg module which is projective over $G\left(\mathbb{F}_{p}\right)$; thus $\left|G\left(\mathbb{F}_{p}\right)\right|_{L(\lambda)}=\{0\}$.

(b) We may now assume that $\Phi_{\lambda}$ is a subroot system of $\Phi$ which is empty or of type $A_{1}$. Let $J=\{\alpha\}$ be the set consisting of one simple root. By [NPV, 6.6.1], we have that $G \cdot \mathfrak{u}_{J} \subseteq|\mathfrak{g}|_{L(\lambda)}$. Note that $\mathfrak{u}_{J}$ is the nilpotent radical of a minimal parabolic subalgebra $P_{\alpha}$. By a result of Steinberg [Hum4, Lemma 5.8 (i)], we have $G \cdot \mathfrak{u}_{\{\alpha\}}=G \cdot \mathfrak{u}_{\{\beta\}}$ for any simple root $\beta$ since $G$ is simple. Thus we can choose the simple root $\alpha$ such that $\mathfrak{u}_{J}$ contains a sufficiently large abelian subalgebra defined over $\mathbb{F}_{p}$. For type $A_{2}, \mathfrak{u}_{J}$ is abelian of dimension 2 , which is also the $p$-rank of the group $G\left(\mathbb{F}_{p}\right)$. For the types $B_{2}$ and $G_{2}$, we can choose $\alpha$ to be the short simple root. Then $\mathfrak{u}_{\alpha}$ contains a three dimensional abelian subalgebra (a sum of three commuting root spaces). Note that $G\left(\mathbb{F}_{p}\right)$ has $p$-rank 3 for both $B_{2}$ and $G_{2}$ (for $p>3$ ). If $J=\varnothing$, then $\mathfrak{u}_{J}$ is the unipotent radical of a Borel subalgebra. An elementary abelian $p$-subgroup of maximal rank is contained in the Borel subgroup which gives an abelian Lie subalgebra of $\mathfrak{g}\left(\mathbb{F}_{p}\right)$ (see Proposition 7 ). Thus $\operatorname{dim}\left|G\left(\mathbb{F}_{p}\right)\right|_{H^{0}(\lambda)}$ is the $p$-rank of the group $G\left(\mathbb{F}_{p}\right)$.

We refer the reader to [GLR, Table 3.3.1] for a list of $p$-ranks of finite simple groups of Lie type.

5.4. Induced modules. In [NPV, (6.2.1) Thm.], it was shown that for $p$ good and $\lambda \in X(T)_{+}$, if $J \subset \Pi$ with $w\left(\Phi_{\lambda}\right)=\Phi_{J}$, then $|\mathfrak{g}|_{H^{0}(\lambda)}=G \cdot \mathfrak{u}_{J}$. The theorem below allows us to use this computation to determine the dimension of support varieties for $H^{0}(\lambda)$ over $G\left(\mathbb{F}_{p}\right)$. The ease of these computations illustrates the power of this geometric approach.

Theorem 9. Let $G$ be a connected reductive algebraic group with $p$ good for $G$. Suppose $\lambda \in X(T)_{+}$such that $\left\langle\lambda, \alpha_{0}^{\vee}\right\rangle<p(p-1) / c$.

(a) If $J^{\prime}$ is a subset of the simple roots such that $\mathfrak{u}_{J^{\prime}}$ is abelian and $\mathfrak{u}_{J^{\prime}} \subseteq$ $|\mathfrak{g}|_{H^{0}(\lambda)}$, then

$$
\operatorname{dim}_{k} \mathfrak{u}_{J^{\prime}} \leq \operatorname{dim}\left|G\left(\mathbb{F}_{p}\right)\right|_{H^{0}(\lambda)} \leq \min \left\{p-\operatorname{rank} G\left(\mathbb{F}_{p}\right), \frac{1}{2} \operatorname{dim}|\mathfrak{g}|_{H^{0}(\lambda)}\right\}
$$

(b) If $w\left(\Phi_{\lambda}\right)=\Phi_{J}$ for some $w \in W$ and $\mathfrak{u}_{J}$ is abelian, then $\operatorname{dim}_{k}\left|G\left(\mathbb{F}_{p}\right)\right|_{H^{0}(\lambda)}=$ $\operatorname{dim}_{k} \mathfrak{u}_{J}$.

(c) If $\Phi_{\lambda}=\varnothing$, then $\operatorname{dim}\left|G\left(\mathbb{F}_{p}\right)\right|_{H^{0}(\lambda)}=\operatorname{dim}\left|G\left(\mathbb{F}_{p}\right)\right|_{k}$.

Proof. The assumption on $\lambda$ insures that $H^{0}(\lambda)$ is in $\mathcal{C}_{p}$. (a) Suppose that $\mathfrak{u}_{J^{\prime}} \subseteq$ $|\mathfrak{g}|_{H^{0}(\lambda)}$ where $\mathfrak{u}_{J^{\prime}}$ is abelian. Then $\mathfrak{u}_{J^{\prime}} \subseteq \mathcal{N}^{\mathbb{F}_{p}} \cap|\mathfrak{g}|_{H^{0}(\lambda)} \subseteq \psi\left(\left|G\left(\mathbb{F}_{p}\right)\right|_{H^{0}(\lambda)}\right)$. Hence, $\operatorname{dim}_{k} \mathfrak{u}_{J^{\prime}} \leq \operatorname{dim}\left|G\left(\mathbb{F}_{p}\right)\right|_{H^{0}(\lambda)}$. The other inequality follows by [LN, Thm. 3.4(b)] and the fact that the dimension of the support variety of a module is always bounded by the dimension of the support variety of the trivial module which is precisely the $p$-rank of the group. 
(b) Under the hypotheses we have $|\mathfrak{g}|_{H^{0}(\lambda)}=G \cdot \mathfrak{u}_{J}$ where $\mathfrak{u}_{J}$ is abelian. Therefore by part (a), $\operatorname{dim}_{k} \mathfrak{u}_{J} \leq \operatorname{dim}\left|G\left(\mathbb{F}_{p}\right)\right|_{H^{0}(\lambda)}$. On the other hand, by [LN, Thm. 3.4(b)], we have

$$
\operatorname{dim}\left|G\left(\mathbb{F}_{p}\right)\right|_{H^{0}(\lambda)} \leq \frac{1}{2} \operatorname{dim}|\mathfrak{g}|_{H^{0}(\lambda)}=\operatorname{dim}_{k} \mathfrak{u}_{J}
$$

The last equality uses the dimension formula (see [C, 5.10.2] and [Hum4, 6.7]).

(c) When $\Phi_{\lambda}=\varnothing$, then the elementary abelian $p$-subgroup of $G\left(\mathbb{F}_{p}\right)$ contained in the unipotent radical of the Borel subgroup $B$ corresponds to an abelian subalgebra of $\mathfrak{g}_{\mathbb{F}_{p}}$ which is contained in $\mathfrak{u}_{J}$ and which has dimension equal to the $p$-rank of the finite group (see Proposition 7).

5.5. Computations for $\mathrm{GL}_{n}$. We now concentrate on the group $G=\mathrm{GL}_{n}$ with underlying root system of type $A_{n-1}$. The $p$-rank of $\mathrm{GL}_{n}\left(\mathbb{F}_{p}\right)$ is given for $n \leq 7$ in the table below.

\begin{tabular}{|l||l|}
\hline group & $p$-rank \\
\hline $\operatorname{GL}_{2}\left(\mathbb{F}_{p}\right)$ & 1 \\
$\operatorname{GL}_{3}\left(\mathbb{F}_{p}\right)$ & 2 \\
$\operatorname{GL}_{4}\left(\mathbb{F}_{p}\right)$ & 4 \\
$\operatorname{GL}_{5}\left(\mathbb{F}_{p}\right)$ & 6 \\
$\operatorname{GL}_{6}\left(\mathbb{F}_{p}\right)$ & 9 \\
$\operatorname{GL}_{7}\left(\mathbb{F}_{p}\right)$ & 12 \\
\hline
\end{tabular}

By using Theorem 9, the dimensions of the support varieties for the induced modules $H^{0}(\lambda)$ with $\lambda \in X_{1}(T)$ can be computed completely for $n \leq 5$. They can also be computed in all but two cases for $n=6$ and $n=7$. These computations are given in the tables below. In the two exceptional cases, the unipotent radical corresponding to the stabilizer $\Phi_{\lambda}$ is not abelian and an elementary abelian $p$-subgroup of largest rank is not contained in the unipotent radical.

\begin{tabular}{|l||l|}
\hline $\mathrm{GL}_{2}\left(\mathbb{F}_{p}\right)$ & \\
\hline$\Phi_{\lambda}$ & $\operatorname{dim}\left|G\left(\mathbb{F}_{p}\right)\right|_{H^{0}(\lambda)}$ \\
\hline$\varnothing$ & 1 \\
$A_{1}$ & 0 \\
\hline
\end{tabular}

\begin{tabular}{|l||l|}
\hline $\mathrm{GL}_{3}\left(\mathbb{F}_{p}\right)$ & \\
\hline$\Phi_{\lambda}$ & $\operatorname{dim}\left|G\left(\mathbb{F}_{p}\right)\right|_{H^{0}(\lambda)}$ \\
\hline$\varnothing$ & 2 \\
$A_{1}$ & 2 \\
$A_{2}$ & 0 \\
\hline
\end{tabular}

\begin{tabular}{|l||l|}
\hline $\mathrm{GL}_{4}\left(\mathbb{F}_{p}\right)$ & \\
\hline$\Phi_{\lambda}$ & $\operatorname{dim}\left|G\left(\mathbb{F}_{p}\right)\right|_{H^{0}(\lambda)}$ \\
\hline$\varnothing$ & 4 \\
$A_{1}$ & 4 \\
$A_{1} \times A_{1}$ & 4 \\
$A_{2}$ & 3 \\
$A_{3}$ & 0 \\
\hline
\end{tabular}

\begin{tabular}{|l||l|}
\hline $\mathrm{GL}_{5}\left(\mathbb{F}_{p}\right)$ & \\
\hline$\Phi_{\lambda}$ & $\operatorname{dim}\left|G\left(\mathbb{F}_{p}\right)\right|_{H^{0}(\lambda)}$ \\
\hline$\varnothing$ & 6 \\
$A_{1}$ & 6 \\
$A_{1} \times A_{1}$ & 6 \\
$A_{2}$ & 6 \\
$A_{2} \times A_{1}$ & 6 \\
$A_{3}$ & 4 \\
$A_{4}$ & 0 \\
\hline
\end{tabular}




\begin{tabular}{|l||l|}
\hline $\mathrm{GL}_{6}\left(\mathbb{F}_{p}\right)$ & \\
\hline$\Phi_{\lambda}$ & $\operatorname{dim}\left|G\left(\mathbb{F}_{p}\right)\right|_{H^{0}(\lambda)}$ \\
\hline$\varnothing$ & 9 \\
$A_{1}$ & 9 \\
$A_{1} \times A_{1}$ & 9 \\
$A_{2}$ & 9 \\
$A_{1} \times A_{1} \times A_{1}$ & 8 or 9 \\
$A_{2} \times A_{1}$ & 9 \\
$A_{3}$ & 8 or 9 \\
$A_{2} \times A_{2}$ & 9 \\
$A_{3} \times A_{1}$ & 8 \\
$A_{4}$ & 5 \\
$A_{5}$ & 0 \\
\hline
\end{tabular}

\begin{tabular}{|l||l|}
\hline $\mathrm{GL}_{7}\left(\mathbb{F}_{p}\right)$ & \\
\hline$\Phi_{\lambda}$ & $\operatorname{dim}\left|G\left(\mathbb{F}_{p}\right)\right|_{H^{0}(\lambda)}$ \\
\hline$\varnothing$ & 12 \\
$A_{1}$ & 12 \\
$A_{1} \times A_{1}$ & 12 \\
$A_{2}$ & 12 \\
$A_{1} \times A_{1} \times A_{1}$ & 12 \\
$A_{2} \times A_{1}$ & 12 \\
$A_{3}$ & 12 \\
$A_{2} \times A_{1} \times A_{1}$ & 12 \\
$A_{2} \times A_{2}$ & 12 \\
$A_{3} \times A_{1}$ & 12 \\
$A_{4}$ & 10 or 11 \\
$A_{3} \times A_{2}$ & 12 \\
$A_{4} \times A_{1}$ & 10 \\
$A_{5}$ & 6 \\
$A_{6}$ & 0 \\
\hline
\end{tabular}

\section{Appendix}

6.1. For a dominant integral weight $\lambda \in X(T)_{+}$, we express $\lambda=\sum_{i=1}^{l} \lambda_{i} \omega_{i}$ in terms of the fundamental weights $\omega_{1}, \omega_{2}, \ldots, \omega_{l}$. We give an estimate for $t^{\vee}\left(\omega_{i}\right)$ for each root system using the table in [Hum1, 13.2] and the fact that $0 \leq t^{\vee}\left(\alpha_{i}\right) \leq 2$ (see Corollary 1).

In this appendix we list all $a_{i}=t^{\vee}\left(\omega_{i}\right)=\sum_{j=1}^{l} 2 b_{i j}$ for $t^{\vee}\left(\alpha_{i}\right)=2$. Hence, $a_{i}$ is twice the sum of the coefficients of $\omega_{i}$ when expressed in terms of simple roots. Set $n_{i}=\left\langle\omega_{i}, \alpha_{0}^{\vee}\right\rangle$ and $b_{i}=a_{i} / n_{i}$. Then $\left\langle\lambda, \alpha_{0}^{\vee}\right\rangle=\sum_{i=1}^{l} n_{i} \lambda_{i}$ and $t^{\vee}(\lambda) \leq$ $\sum_{i=1}^{l} b_{i} n_{i} \lambda_{i} \leq c\left\langle\lambda, \alpha_{0}^{\vee}\right\rangle$, where $c=\max _{i}\left\{b_{i}\right\}$. The Coxeter number $h$ is given by the formula $h=1+\sum_{i=1}^{l} n_{i}$.

\begin{tabular}{|c|c|c|c|}
\hline Type & $\left(n_{1}, \ldots, n_{l}\right)$ & $h$ & $\mathrm{c}$ \\
\hline$A_{l}$ & $(1,1, \ldots, 1)$ & $l+1$ & $((l+1) / 2)^{2}$ \\
\hline$B_{l}$ & $(2,2, \ldots, 2,1)$ & $2 l$ & $l(l+1) / 2$ \\
\hline$C_{l}$ & $(1,2, \ldots, 2)$ & $2 l$ & $l^{2} / 2$ \\
\hline$D_{l}$ & $(1,2, \ldots, 2,1,1)$ & $2 l-2$ & $l(l-1) / 2$ \\
\hline$E_{6}$ & $(1,2,2,3,2,1)$ & 12 & 16 \\
\hline$E_{7}$ & $(2,2,3,4,3,2,1)$ & 18 & 27 \\
\hline$E_{8}$ & $(2,3,4,6,5,4,3,2)$ & 30 & 46 \\
\hline$F_{4}$ & $(2,4,3,2)$ & 12 & 11 \\
\hline$G_{2}$ & $(2,3)$ & 6 & 4 \\
\hline
\end{tabular}

6.2. The quantity $a_{i}$ is given below for all indecomposable root systems.

Type A. For $i=1,2, \ldots, l$,

$$
a_{i}=\frac{2}{l+1}\left[(l-i+1) \sum_{j=1}^{i} j+i \sum_{j=i+1}^{l}(l-j+1)\right]=i(l-i+1),
$$

(6.2.2) $\quad c=(l+1)^{2} / 4$. 
Type B. For $i=1,2, \ldots, l-1$,

$$
\begin{aligned}
a_{i} & =\sum_{j=1}^{i} 2 j+i \sum_{j=i+1}^{l} 2=i(2 l-i+1) \leq l(l+1), \\
a_{l} & =\frac{1}{2} \sum_{j=1}^{l} 2 j=l(l+1) / 2, \\
c & =l(l+1) / 2 .
\end{aligned}
$$

Type C. For $i=1,2, \ldots, l-1$,

$$
\begin{aligned}
a_{i} & =\sum_{j=1}^{i-1} 2 j+i(2+\cdots+2+1)=i(2 l-i), \\
c & = \begin{cases}l^{2} / 2 & \text { if } l>3, \\
5 & \text { if } l=3 .\end{cases}
\end{aligned}
$$

Type D. For $i=1,2, \ldots, l-2$,

$$
\begin{aligned}
& a_{i}=\sum_{j=1}^{i} 2 j+i(2+\cdots+2+1+1)=i(2 l-3-i), \\
& a_{l-1}=a_{l} \leq(1+2+\cdots+(l-2))+2 l-2=l(l-1) / 2, \\
& \text { (6.2.10) } \quad c=l(l-1) / 2 \quad(l \geq 4) \text {. }
\end{aligned}
$$

Exceptional groups.

\begin{tabular}{|c|c|c|c|c|c|c|c|c|c|c|}
\hline \multirow{2}{*}{$\begin{array}{c}\text { Index } \\
i\end{array}$} & \multicolumn{2}{|c|}{ Type $E_{6}$} & \multicolumn{2}{|c|}{ Type $E_{7}$} & \multicolumn{2}{|c|}{ Type $E_{8}$} & \multicolumn{2}{|c|}{ Type $F_{4}$} & \multicolumn{2}{c|}{ Type $G_{2}$} \\
\hline & $a_{i}$ & $b_{i}$ & $a_{i}$ & $b_{i}$ & $a_{i}$ & $b_{i}$ & $a_{i}$ & $b_{i}$ & $a_{i}$ & $b_{i}$ \\
\hline 1 & 16 & 16 & 34 & 17 & 92 & 46 & 22 & 11 & 6 & 3 \\
\hline 2 & 22 & 11 & 49 & 25 & 136 & 46 & 42 & 11 & 10 & 4 \\
\hline 3 & 30 & 15 & 66 & 22 & 182 & 26 & 30 & 10 & & \\
\hline 4 & 42 & 12 & 96 & 24 & 270 & 45 & 16 & 8 & & \\
\hline 5 & 30 & 15 & 75 & 25 & 220 & 44 & & & & \\
\hline 6 & 16 & 16 & 52 & 26 & 168 & 42 & & & & \\
\hline 7 & & & 27 & 27 & 114 & 38 & & & & \\
\hline 8 & & & & & 58 & 29 & & & & \\
\hline
\end{tabular}

\section{REFERENCES}

[A] J.L. Alperin, Diagrams for modules, J. Pure and Appl. Algebra 16 (1980), 111-119. MR556154 (81h:16047)

[AM1] J.L. Alperin, G. Mason, On simple modules for SL(2,q), Bull. London Math. Soc. 25 (1993), no. 1, 17-22. MR1190358 (93j:20033)

[AM2] J.L. Alperin, G. Mason, Partial Steinberg modules for finite groups of Lie type, Bull. London Math. Soc. 25 (1993), no. 6, 553-557. MR1245081 (94i:20024)

[AJ] H. H. Andersen, J. C. Jantzen, Cohomology of induced representations for algebraic groups, Math. Ann. 269, (1984), 487-525. MR766011 (86g:20057)

[AS] G.S. Avrunin, L.L. Scott, Quillen stratification for modules, Invent. Math. 66 (1982), 277-286. MR656624 (83h:20048)

[ABS] H. Azad, M. Barry, G. Seitz, On the structure of parabolic groups, Comm. Algebra 18 (2) (1990), 551-562. MR1047327 (91d:20048)

[Ben1] D.J. Benson, Representations and cohomology. II. Cohomology of groups and modules. Cambridge Studies in Advanced Mathematics, 31. Cambridge University Press, Cambridge, 1991. MR1156302 (93g:20099) 
[Ben2] D.J. Benson, Modular representation theory: new trends and methods. Lecture Notes in Mathematics, 1081. Springer-Verlag, Berlin, 1984. MR765858 (86g:20013)

[Bo] A. Borel, Linear Algebraic Groups, Second Enlarged Edition, Springer-Verlag, New York, 1991. MR1102012 (92d:20001)

[B] N. Bourbaki, Groupes et algèbres de Lie, 4-6, Hermann, 1968. MR0240238 (39:1590)

[CLNP] J.F. Carlson, Z. Lin, D.K. Nakano, B.J. Parshall, The restricted nullcone, Contemp. Math., 325 (2003), 51-75. MR1988985 (2004g:17017)

[C] R.W. Carter, Finite groups of Lie type, Wiley-Interscience, 1985. MR794307 (87d:20060)

[Ch] L.G. Chouinard, Projectivity and relative projectivity over group rings. J. Pure Appl. Algebra 7 (1976), no. 3, 287-302. MR0401943 (53:5769)

[CPSvdK] E. Cline, B. Parshall, L. Scott, W. van der Kallen, Rational and generic cohomology Invent. Math. 39 (1977), 143-163. MR0439856 (55:12737)

[CM] D.H. Collingwood, W.M. McGovern, Nilpotent Orbits in Semisimple Lie Algebras, Van Nostrand Reinhold, 1993. MR1251060 (94j:17001)

[D] S. Donkin, The normality of closures of conjugacy classes of matrices, Invent. Math. 101 (1990), 717-736 MR1062803 (91j:14040)

[FP1] E.M. Friedlander, B.J. Parshall, On the cohomology of algebraic and related finite groups, Invent. Math. 74 (1983), 85-117. MR722727 (85d:20035)

[FP2] E.M. Friedlander, B.J. Parshall, Support varieties for restricted Lie algebras, Invent. Math. 86 (1986), 553-562. MR860682 (88f:17018)

[FPe] E.M. Friedlander, J. Pevtsova, Representation theoretic support spaces for finite group schemes, Amer. J. Math 127 (2005), 379-420. MR2130619 (2005k:14096)

[FS] E.M. Friedlander, A.A. Suslin, Cohomology of finite group schemes over a field, Invent. Math. 127 (1997), no. 2, 209-270. MR1427618 (98h:14055a)

[GLR] D. Gorenstein, R. Lyons, R. Solomon, The classification of the finite simple groups, Number 3. Part I. Chapter A, Almost simple K-groups. Mathematical Surveys and Monographs, 40.3. Amer. Math. Soc., Providence, 1998. MR1490581 (98j:20011)

[HS] D.F. Holt, N. Spaltenstein, Nilpotent orbits of exceptional Lie algebras over algebraically closed fields of bad characteristics, J. Australian Math. Soc. Ser. A 38 (1985), 330-350. MR779199 (86g:17007)

[Hum1] J.E. Humphreys, Introduction to Lie Algebras and Representation Theory, SpringerVerlag, 1972. MR0323842 (48:2197)

[Hum2] J.E. Humphreys, Linear Algebraic Groups, Springer-Verlag, 1975. MR0396773 (53:633)

[Hum3] J.E. Humphreys, Ordinary and Modular Representations of Chevalley Groups, Lecture Notes in Math. 528, Springer-Verlag, 1976. MR0453884 (56:12137)

[Hum4] J.E. Humphreys, Conjugacy Classes in Semisimple Algebraic Groups, Mathematical Surveys and Monographs 43, Amer. Math. Soc., Providence, 1995. MR1343976 (97i:20057)

[JJ] I. Janiszczak, J.C. Jantzen, Simple periodic modules over Chevalley groups, J. London Math. Soc. 41 (1990), 217-230. MR1067263 (91f:20010)

[Jan1] J.C. Jantzen, Representations of Algebraic Groups, Academic Press, 1987. MR899071 (89c:20001)

[Jan2] J.C. Jantzen, Representations of Chevalley groups in their own characteristic. The Arcata Conference on Representations of Finite Groups (Arcata, Calif., 1986), 127146, Proc. Sympos. Pure Math., 47, Part 1, Amer. Math. Soc., Providence, RI, 1987. MR933356 (89g:20076)

[Jan3] J.C. Jantzen, Support varieties of Weyl modules, Bull. London Math. Soc. 19 (1987), 238-244. MR879510 (88e:17008)

[Jan4] J.C. Jantzen, Nilpotent Orbits in Representation Theory, Progr. Math. 228, Birkhäuser, Boston, 2004.

[KLT] S. Kumar, N. Lauritzen, J.F. Thomsen, Frobenius splitting of cotangent bundles of flag varieties, Invent. Math. 136 (1999), 603-621. MR1695207 (2000g:20088)

[LN] Z. Lin, D.K. Nakano, Complexity for modules over finite Chevalley groups and classical Lie algebras, Invent. Math. 138 (1999), 85-101. MR1714337 (2000m:20077)

[LT] R. Lawther, D. Testerman, $A_{1}$-subgroups of exceptional algebraic groups, Memoirs of Amer. Math. Soc. No. 674 (1999). 
[McN1] G.J. McNinch, Abelian unipotent subgroups of reductive groups, J. Pure and Applied Algebra 167 (2002), 269-300. MR1874545 (2002i:20064)

[McN2] G.J. McNinch, Optimal SL(2)-Homomorphisms, Comment. Math. Helv. 80 (2005), 391-426. MR2142248 (2006f:20055)

[McN3] G.J. McNinch, Sub-principal homomorphisms in positive characteristics, Math. Z. 244 (2003), 433-455. MR1992546 (2004c:20080)

[NPV] D.K. Nakano, B.J. Parshall, D.C. Vella, Support varieties for algebraic groups, J. Reine Angew. Math. 547 (2002), 15-47. MR1900135 (2003b:20063)

[P] B.J. Parshall, Cohomology of algebraic groups, Proc. Symp. Pure Math., 47 (1987), 233-248. MR933362 (89b:20095)

[PV] V.L. Popov, E.B. Vinberg, Invariant theory, Algebraic Geometry IV (Encyclopaedia of Math. Sci. Vol. 55), Springer-Verlag, 1994, 123-278.

[Sei] G. Seitz, Unipotent elements, tilting modules, and saturation, Invent. Math. 141 (2000) 3, 467-502. MR1779618 (2001j:20074)

[Ser] J.P. Serre, Lie Algebras and Lie Groups, LNM 1500, Springer-Verlag, 1992. MR1176100 (93h:17001)

[Sh] I. Shafarevich, Basic Algebraic Geometry, Vol. 1, 2nd Edition, Springer-Verlag, Berlin/Heidelberg, 1994.

[Sp1] T.A. Springer, The unipotent variety of a semisimple group, Proc. Coll. in Alg. Geom. (Tata Institute) (1969), 373-391. MR0263830 (41:8429)

[Sp2] T.A. Springer, Linear Algebraic Groups, Progress in Math. 9, Birkhäuser, 1998. MR1642713 (99h:20075)

[Sp3] T.A. Springer, Linear algebraic groups, Algebraic Geometry IV (Encyclopaedia of Math. Sci.) Vol. 55, Springer-Verlag, (1994), 1-121. MR1309681 (95g:14002)

[SS] T.A. Springer, R. Steinberg, Conjugacy Classes, Seminar on Algebraic Groups and Related Finite Groups, LNM 131, Springer-Verlag, (1970) 167-266. MR0268192 (42:3091)

[St] R. Steinberg, Lectures on Chevalley Groups, Yale University, 1967. MR0466335 (57:6215)

[SFB] A. Suslin, E. Friedlander, C. Bendel, Support varieties for infinitesimal group schemes, Journal of AMS 10, (1997), 729-759. MR1443547 (98h:14055c)

[T1] D. M. Testerman, The construction of the maximal $A_{1}$ 's in the exceptional algebraic groups, Proc. Amer. Math. Soc., 116 (1992), 635-644. MR1100666 (93a:20073)

[T2] D. M. Testerman, $A_{1}$-type overgroups of elements of order $p$ in semisimple algebraic groups and the associated finite groups. J. Algebra 177 (1995), 34-76. MR1356359 (96j:20067)

[Th] J.F. Thomsen, Normality of certain nilpotent varieties in positive characteristic, $J$. Algebra 226 (2000), 865-874. MR1759837 (2001g:14079)

[UGA] University of Georgia VIGRE Algebra Group, Varieties of nilpotent elements of simple Lie algebras I: good primes, J. Algebra 280 (2004), 719-737. MR2090060 (2005h:17016)

Department of Mathematics, University of Georgia, Athens, Georgia 30602

E-mail address: jfc@math.uga.edu

Department of Mathematics, Kansas State University, Manhattan, Kansas 66506

E-mail address: zlin@math.ksu.edu

Department of Mathematics, University of Georgia, Athens, Georgia 30602

E-mail address: nakano@math.uga.edu 